\title{
Local Definiteness, Primarity and Quasiequivalence of Quasifree Hadamard Quantum States in Curved Spacetime
}

\author{
Rainer Verch $\operatorname{Vi}^{\star} \star \star$ \\ Institut für Theoretische Physik, Freie Universität Berlin, Berlin, Germany
}

Received: 31 August 1992/in revised form: 24 August 1993

\begin{abstract}
We prove that the GNS-representations of quasifree, Hadamard states on the Weyl-algebra of the quantized Klein-Gordon field propagating in an arbitrary globally hyperbolic spacetime are locally quasiequivalent. We also show that these representations satisfy local primarity and local definiteness if the spacetime is assumed to be ultrastatic. This implies that the local von Neumann algebras associated with these representations are type $I I I_{1}$-factors for sufficiently small regions in ultrastatic spacetimes.
\end{abstract}

\section{Introduction}

Since Hawking's remarkable paper in 1975 [Haw] on the particle creation by black holes due to quantum effects, significant progress in the mathematical foundations of quantum field theory in curved spacetime has been made. In particular, it has been realized that a very concise and mathematically rigorous description of quantum field theory in curved spacetime may be reached by the methods of algebraic quantum field theory. These methods have already been successfully applied in the case of linear fields propagating in the background of globally hyperbolic spacetimes and have given important insights for instance in the interplay of the geometrical structure of the spacetime background and the algebraic structure of the theory (see e.g. [Ban; Dim; FH1,2; H1,2; HNS; Kay1,3,5; KW; LR] and references cited therein).

Still, there remains the question as to which states, if any, play the role of the "vacuum" state as in the Minkowski-spacetime quantum field theory in selecting the set of "physically relevant states." To clarify what is meant by that, recall that in algebraic quantum field theory the description of a system (e.g. a quantum field propagating in a fixed spacetime background) is given through the specification of a

\footnotetext{
* Supported by the DFG, SFB 288 "Differentialgeometrie und Quantenphysik"

$\star \star$ Present address: II. Inst. f. Theor. Phys., Universität Hamburg, Luruper Chaussee 149, D-22761 Hamburg, e-mail: i02ver@dhhdesy3.bitnet
} 
pair $(\mathcal{A}, \mathcal{S})$, where $\mathcal{A}$ is the algebra of quasilocal observables of the system, arising (in the case of interest for us) as the $C^{*}$-inductive limit of a net $\mathcal{O} \mapsto \mathcal{A}(\mathcal{O})$ of $C^{*}$ algebras which constitute the algebras of local observables which can be measured within finite spacetime regions $\mathcal{O}$ (i.e. $\mathcal{O}$ is an open subset of the spacetime-manifold with compact closure). $\mathcal{S}$ is the set of all "physically relevant states" of the system and is a specified subset of the set $\mathcal{A}_{1}^{*+}$ of all positive, linear, normalized functionals on $\mathcal{A}$.

On grounds of principle one expects that $\mathcal{S}$ should be specified in such a way that the principles of local definiteness, local primarity and local quasiequivalence (or local normality) are satisfied, which we formulate as follows with the convention that $\pi_{\omega}$ denotes the GNS-representation of $\omega \in \mathcal{S}$, and $\mathcal{R}_{\omega}(\mathcal{O}):=\pi_{\omega}(\mathcal{A}(\mathcal{O}))^{\prime \prime}$.

Definition 1.1. For the description $(\mathcal{A}, \mathcal{S})$ of a physical system, we define:

1. Local Definiteness: $\bigcap_{\mathcal{O}} \mathcal{R}_{\omega}(\mathcal{O})=\mathbf{C 1}$ for all points $p$ in the spacetime and $\omega \in \mathcal{S}$.

2. Local Primarity: $\mathcal{R}_{\omega}(\mathcal{O})$ is a factor for all $\omega \in \mathcal{S}$.

3. Local Quasiequivalence: $\pi_{\omega}$ and $\pi_{\hat{\omega}}$ are locally quasiequivalent for all $\omega, \hat{\omega} \in \mathcal{S}$.

We give a few brief comments on these requirements, for further discussion see the references [H1; HNS; Kay5; KW; LR] and, in particular, the recent book by Haag [H2]. There appears to be some overlap concerning the motivation: Local definiteness and local quasiequivalence imply that there are no nontrivial measurements of vanishing spatio-temporal extension on $\omega \in \mathcal{S}$, which corresponds to the idea that physical states should have a finite spatio-temporal energy-density. Consequently, physical states cannot be distinguished by measurements at single spacetime points. Local primarity means that there are no macroscopic, or classical, observables which could be measured within finite spacetime regions on $\omega \in \mathcal{S}$. This is clearly expected fur sufficiently small spacetime regions $\mathcal{O}$, by the experience that in the small the world obeys "quantum laws." Similarly, the condition of local quasiequivalence rules out the possibility of local superselection rules, which are expected to be phenomena of global or topological nature.

We have not given a precise statement of what we mean by "local quasiequivalence." There are at least two reasonable interpretations of that (see the footnote on p. 70 of $[\mathrm{KW}]$ ) which are, as has already been remarked in [LR], equivalent in the presence of local primarity. What we mean by local quasiequivalence is the following: Given an open spacetime region $\mathcal{O}$ with compact closure, $\pi_{\omega} \mid \mathcal{A}(\mathcal{O})$ and $\pi_{\hat{\omega}} \mid \mathcal{A}(\mathcal{O})$ are quasiequivalent, that is, there is an isomorphism $\beta_{\mathcal{O}}: \mathcal{R}_{\omega}(\mathcal{O}) \rightarrow \mathcal{R}_{\hat{\omega}}(\mathcal{O})$ such that $\beta_{\mathcal{O}} \circ \pi_{\omega}(A)=\pi_{\hat{\omega}}(A)$ for all $A \in \mathcal{A}(\mathcal{O})$.

It is also expected that $\mathcal{S}$ forms a convex subset in $\mathcal{A}_{1}^{*+}$. This is automatically satisfied if we assume that $\mathcal{S}$ is maximal in the sense that if some state $\omega_{0}$ is in $\mathcal{S}$, then all states $\omega$ for which $\pi_{\omega}$ is locally quasiequivalent to $\pi_{\omega_{0}}$ are also in $\mathcal{S}$. (If $\omega_{0}$ satisfies conditions 1 and 2 of Def. 1.1, then so do all $\omega$ thus obtained by local quasiequivalence, hence the assumption is consistent.) Then $\mathcal{S}$ is the set of all $\omega \in \mathcal{A}_{1}^{*+}$ lying locally in the folium of $\omega_{0}$, i.e. for a spacetime region $\mathcal{O}$ with compact closure there is a density matrix $\varrho_{\mathcal{O}, \omega}$ on the GNS-Hilbertspace of $\omega_{0}$ such that $\omega(A)=\operatorname{tr}\left(\varrho_{\mathcal{O}, \omega} \pi_{\omega_{0}}(A)\right)$ for all $A \in \mathcal{A}(\mathcal{O})$. In this situation $\omega_{0}$ determines an equivalence class, $\mathcal{S}$, in $\mathcal{A}_{1}^{*+}$ with respect to the equivalence relation "local quasiequivalence."

For Minkowski-spacetime, there is an obvious choice for a preferred physical state $\omega_{0}$ : The vacuum, which is mainly characterized by the invariance under Poincarétransformations and the spectral condition. But on a generic spacetime we do not 
have symmetries that could likewise serve to single out a distinguished state as the vacuum. However, there might be classes of certain preferred states which are regarded as physical states, somehow selected and specified. If $\mathcal{S}_{0} \subset \mathcal{A}_{1}^{*+}$ is such a class of preferred states, then the question is if all $\omega_{0} \in \mathcal{S}_{0}$ define the same local quasiequivalence class $\mathcal{S}$ of states, and if they satisfy 1 and 2 above. Provided this is the case, one may choose the set consisting of all the states lying locally in the folia of the states in $\mathcal{S}_{0}$ as the set of all physical states. So, if one has made a choice of $\mathcal{S}_{0}$, one must check if the requirements of Definition 1.1 hold for all $\omega, \hat{\omega} \in \mathcal{S}_{0}$.

Now we take a look at three prominent candidates for classes $\mathcal{S}_{0}$ in the case that our system is the Klein-Gordon field propagating in some spacetime $(\mathcal{M}, g)$. These are

(a) $\mathcal{S}_{0}=$ set of all quasifree states possessing scaling limits at all spacetime points and satisfying local stability,

(b) $\mathcal{S}_{0}=$ set of all adiabatic vacuum states,

(c) $\mathcal{S}_{0}=$ set of all quasifree Hadamard states.

Let us give brief comments on these classes of states.

(a) The notion of "local stability," which roughly amounts to a suitable local replacement of the spectral condition, has been introduced by Haag, Narnhofer and Stein [HNS] (to which we refer for full details, see also [FH1; H2]). These authors seem to have first formulated the requirements of Definition 1.1, which they refer to as conditions for what they call "principle of local definiteness." Essentially, they show that local definiteness implies both that scaling limits are those of the free, massless theory in flat spacetime, and local stability. However, they also show that the scaling limit assumption together with local stability is not sufficient to guarantee local quasiequivalence.

(b) An "adiabatic vacuum" (see [LR] and references therein for full details) is a state which satisfies a suitable condition restricting its high frequency behaviour. Lüders and Roberts [LR] proved that all adiabatic vacuum states on the algebra of quasilocal observables of the Klein-Gordon field in Robertson-Walker spacetimes are locally quasiequivalent and locally primary.

(c) We shall quote the full definition of "Hadamard state" (due to [KW]) and list a number of relevant properties in Appendix C. Suffice it to say here that the Hadamard condition amounts to a certain condition on the short distance behaviour of a quantum state. (We note that (b) and (c) are more restrictive than (a). We also note that adiabatic vacuum states can only be defined for a restricted class of spacetimes (e.g. RobertsonWalker spacetimes) and thus the Hadamard condition is more general. However, the relationship between Hadamard states and adiabatic vacuum states (when the latter can be defined) is not fully investigated yet. See [LR] and literature cited there for further discussion.) We shall also give some very brief comments on the motivation of (c). Apparently, the main motivation to consider Hadamard states originated from the wish to obtain a reasonable expression for the (expectation value of the) energy-momentumtensor of quantum fields in generic spacetimes. This, in turn, was motivated by the attempt to gain a more complete description of gravity incorporating the "backreaction"-effects of particles created in strong gravitational fields and thus to arrive at a "semiclassical" description of gravity whose central equation is the "backreactionequation" or "generalized semiclassical Einstein equation,"

$$
G_{\mu \nu}=8 \pi\left\langle T_{\mu \nu}\right\rangle_{\omega}
$$


(see e.g. [Wal1]). Wald formulated a set of axioms for $\left\langle T_{\mu \nu}\right\rangle_{\omega}$ so as to make it an object giving reasonable physics and to make it "as independent as possible" from the renormalization procedures necessary for its definition. It turned out that for the KleinGordon field in globally hyperbolic spacetimes, $\left\langle T_{\mu \nu}\right\rangle_{\omega}$ can be defined for Hadamard states $\omega$ such that Wald's axioms are fulfilled. The status of the ambiguity that Wald's axioms still allow for $\left\langle T_{\mu \nu}\right\rangle_{\omega}$ has been source of some discussion and it seems that this question has not reached a final answer yet (see [Ful; Kay5; KW; Wal1,2] for further discussion). However, what we just remarked should explain the interest in Hadamard states and the reason why they are assumed to be "physically relevant." In fact, a number of important investigations concerning the properties of Hadamard states have been carried out ([FNW; FSW; GK; KW; Rad] are only a few significant references). It should, however, be remarked that a fully satisfactory definition of Hadamard states was first arrived at in the article by Kay and Wald [KW].

The results of the present work are as follows. We prove that the GNSrepresentations of any two quasifree Hadamard states on the Weyl-algebra of the massive Klein-Gordon field in an arbitrary globally hyperbolic spacetime are locally quasiequivalent. That this should hold has already been conjectured in 1979 by Kay [Kay2] and has been re-conjectured a couple of times since [GK; Kay5] (cf. also [H2]). We also show that the states of the just described type are locally primary and definite (i.e. they satisfy 1 and 2 of Definition 1.1) for ultrastatic, globally hyperbolic spacetimes (local primarity then holds for a class of regions $\mathcal{O}$ forming a base of the spacetime topology). Further, we can use a result by Wollenberg [Wol] to show that in the ultrastatic situation, $\mathcal{R}_{\omega}(\mathcal{O})$ is a factor of type $I I I_{1}$ for all quasifree Hadamard states of the Klein-Gordon field and sufficiently small regions $\mathcal{O}$.

This work is organized in the following way. In the second section we summarize basic material about the Klein-Gordon field in globally hyperbolic spacetimes. In Sect. 3 we present the quasiequivalence result. In Sect. 4 we establish the results on local primarity and local definiteness in ultrastatic spacetimes. Some technical details will be relegated to the Appendix.

\section{The Klein-Gordon Field in Globally Hyperbolic Spacetimes}

We start our discussion by recalling that a globally hyperbolic spacetime is a pair $(\mathcal{M}, g)$ which is a four-dimensional, time-oriented Lorentz-manifold with signature (+- - ) (note that we understand a manifold to be orientable, connected, paracompact, smooth and Hausdorff, without boundary) possessing a smooth foliation into smooth, spacelike Cauchy-surfaces (see [Die]): This means that there is a diffeomorphism $F: \mathbf{R} \times M \rightarrow \mathcal{M}$, where $M$ is a smooth, three-dimensional manifold such that for all $t \in \mathbf{R}, M_{t}:=F(\{t\} \times M)$ is a spacelike Cauchy-surface for $(\mathcal{M}, g)$. Then $\left\{\tau_{t}\right\}, t \in \mathbf{R}$, defined by $\tau_{t}: F\left(t_{0}, x\right) \mapsto F\left(t_{0}+t, x\right)$ gives rise to a one-parametric group of diffeomorphisms of $\mathcal{M}$ which, however, need not be isometries of $(\mathcal{M}, g)$. But there are classes of spacetimes where this is the case, and we shall define a very special class of those, namely the ultrastatic spacetimes.

Definition 2.1. Let $(M, \gamma)$ be a smooth, three-dimensional Riemannian manifold, and let $\left(x^{i}\right)$ be a local coordinate system for $M$. Define $\mathcal{M}:=\mathbf{R} \times M$ and define $a$ Lorentzian metric $g$ on $\mathcal{M}$ by setting the coordinate expression of $g$ in the chart $\left(t, x^{2}\right)$ equal to $d t^{2}-\gamma_{i j} d x^{i} d x^{j}$. We call this $(\mathcal{M}, g)$ the ultrastatic spacetime foliated by $(M, \gamma)$, and $M_{t}:=\{t\} \times M, t \in \mathbf{R}$ the natural foliation of $\mathcal{M}$ (in Cauchy-surfaces, if each $M_{t}$ is a Cauchy-surface). 
One can easily show that the vector field $\xi^{a}=\left(\partial_{t}\right)^{a}$ is a timelike Killing vectorfield which is orthogonal to the hypersurfaces $M_{t}$. Moreover, one can show that the $M_{t}$ are spacelike Cauchy-surfaces for $(\mathcal{M}, g)$ if and only if $(M, \gamma)$ is a complete Riemannian manifold, in which case the ultrastatic spacetime foliated by $(M, \gamma)$ is globally hyperbolic [Kay1]. This situation will be of interest for us later on.

A consequence of global hyperbolicity is the well-posedness of the Cauchyproblem for hyperbolic differential equations. To ease our way of speaking, we shall from now on always assume that our Cauchy-surfaces are spacelike. We shall consider the Klein-Gordon equation (hereafter referred to as KG-Eq.) on a globally hyperbolic spacetime $(\mathcal{M}, g)$,

$$
\left(\nabla^{a} \nabla_{a}+m^{2}\right) \varphi=0
$$

where $m$ is a positive constant, and $\nabla$ is the Levi-Civita connection of $g$.

From now on, all spaces of functions, like $\mathcal{D}(\mathcal{M}), L^{2}(\mathcal{M}, d \eta)$ etc. are meant to be spaces of real-valued functions unless otherwise indicated (by a subscript $\mathbf{C}$ ) where $\mathcal{D}(\mathcal{M})=C_{0}^{\infty}(\mathcal{M}), \mathcal{E}(\mathcal{M})=C^{\infty}(\mathcal{M})$ are spaces of testfunctions endowed with locally convex topologies as defined in [D]. Important sets of causal dependence of subsets $\mathcal{O}$ of $\mathcal{M}$ are $J^{ \pm}(\mathcal{O})=$ set of all points $p \in \mathcal{M}$ which can be reached by future $(+) /$ past $(-)$-directed causal curves emanating from $\mathcal{O}$ (called the causal future/past of $\mathcal{O}), J(\mathcal{O})=J^{+}(\mathcal{O}) \cup J^{-}(\mathcal{O}), D^{ \pm}(\mathcal{O})=$ set of all points $p \in J^{ \pm}(\mathcal{O})$ such that every past $(+) /$ future $(-)$-inextendible causal curve starting at $p$ passes through $\mathcal{O}$, and $D(\mathcal{O})=D^{+}(\mathcal{O}) \cup D^{-}(\mathcal{O})$ (called the domain of dependence of $\mathcal{O}$, or the diamond based at $\mathcal{O}$ ). See [Wal3] for further discussion. Notice that we shall also define $\diamond(\mathcal{O}):=\operatorname{int}(D(\mathcal{O}))$.

The well-posedness of the Cauchy-problem for the KG-Eq. (2.1) has the consequence that there are two unique continuous linear maps $E^{ \pm}: \mathcal{D}(\mathcal{M}) \rightarrow \mathcal{E}(\mathcal{M})$ with the property

$$
\left(\nabla^{a} \nabla_{a}+m^{2}\right) E^{ \pm} f=f=E^{ \pm}\left(\nabla^{a} \nabla_{a}+m^{2}\right) f
$$

for all $f \in \mathcal{D}(\mathcal{M})$ and

$$
\operatorname{supp}\left(E^{ \pm} f\right) \subset J^{ \pm}(\operatorname{supp}(f))
$$

(see $[\mathrm{Dim}])$. They are called the $\operatorname{advanced}(+)$ and $\operatorname{retarded}(-)$ fundamental solutions of the KG-Eq. (2.1). $E:=E^{+}-E^{-}$is called the propagator of the KG-Eq., and by (2.2) it follows that

$$
\left(\nabla^{a} \nabla_{a}+m^{2}\right) E f=0=E\left(\nabla^{a} \nabla_{a}+m^{2}\right) f
$$

for all $f \in \mathcal{D}(\mathcal{M})$. So $E$ maps $\mathcal{D}(\mathcal{M})$, by (2.3), to the set of smooth solutions of (2.1) which have compact support on Cauchy-surfaces (and one can show that this map is surjective, see [Dim]). Now let $\mathcal{C}$ be any Cauchy-surface of $(\mathcal{M}, g)$ and let $\mathcal{C}_{*}$ be an open subset of $\mathcal{C}$. Define $\mathbf{D}_{\mathcal{C}_{*}}:=\mathcal{D}\left(\mathcal{C}_{*}\right) \oplus \mathcal{D}\left(\mathcal{C}_{*}\right)$ and

$$
\delta_{\mathcal{C}_{*}}\left(\left(u_{0}, u_{1}\right),\left(w_{0}, w_{1}\right)\right):=\int_{\mathcal{C}}\left(u_{0} w_{1}-u_{1} w_{0}\right) d \eta_{\mathcal{C}},
$$

where $d \eta_{\mathcal{C}}$ is the induced measure on $\mathcal{C}$; it is easy to see that $\delta_{\mathcal{C}_{*}}$ is a symplectic form on $\mathbf{D}_{\mathcal{C}_{*}}$. Now let $K:=\mathcal{D}(\mathcal{M}) / \operatorname{ker} E$, and [.] $: \mathcal{D}(\mathcal{M}) \rightarrow K$ the quotient map. Further, let $\diamond\left(\mathcal{C}_{*}\right)=\operatorname{int}\left(\mathcal{D}\left(\mathcal{C}_{*}\right)\right)$ be the open interior of the diamond based at $\mathcal{C}_{*}$ and define $K_{\mathcal{C}_{*}}:=\left[\mathcal{D}\left(\diamond\left(\mathcal{C}_{*}\right)\right)\right]$. We define for $[f],[h] \in K_{\mathcal{C}_{*}}$

$$
\kappa_{\mathcal{C}_{*}}([f],[h]):=\int_{\mathcal{M}} f E h d \eta
$$


$d \eta$ denoting the metric-induced measure on $\mathcal{M}$. If $\mathcal{C}_{*}$ is itself a Cauchy-surface, we will simply write $\kappa$ for $\kappa_{\mathcal{C}_{*}}$, since it is independent of the choice of the Cauchy-surface $\mathcal{C}$. The following proposition can be inferred from Lemmas A 1 and A 3 in [Dim].

Proposition 2.2. (a) $\left(\mathbf{D}_{\mathcal{C}_{*}}, \delta_{\mathcal{C}_{*}}\right)$ and $\left(K_{\mathcal{C}_{*}}, \kappa_{\mathcal{C}_{*}}\right)$ are symplectomorphic symplectic spaces. The symplectomorphism

$$
T_{\mathcal{C}_{*}}:\left(K_{\mathcal{C}_{*}}, \kappa_{\mathcal{C}_{*}}\right) \rightarrow\left(\mathbf{D}_{\mathcal{C}_{*}}, \delta_{\mathcal{C}_{*}}\right)
$$

is given by

$$
T_{\mathcal{C}_{*}}([f])=\left(E f\left|\mathcal{C}, n^{a} \nabla_{a} E f\right| \mathcal{C}\right),
$$

where $n^{a}$ is the future-directed unit-normalfield on $\mathcal{C}$.

(b) If $\mathcal{N}$ is any open neighbourhood, in $\mathcal{M}$, of $\mathcal{C}_{*}$, then $K_{\mathcal{C}_{*}} \subset[\mathcal{D}(\mathcal{N})]$.

To a symplectic space $(Z, \zeta)$ there is associated (uniquely up to $C^{*}$-algebraic equivalence) its Weyl-algebra $\mathcal{A}[Z, \zeta]$, which is a simple $C^{*}$-algebra generated by a family $W(z), z \in Z$, of unitary elements satisfying the Weyl-representations of the CCR,

$$
W(z) W(\tilde{z})=e^{-\frac{i}{2} \zeta(z, \tilde{z})} W(z+\tilde{z})
$$

for all $z, \tilde{z} \in Z$. For the symplectic space $(Z, \zeta)=(K, \kappa)$, we refer to $\mathcal{A}[K, \kappa]$ as the Weyl-algebra of the KG-field in the globally hyperbolic spacetime $(\mathcal{M}, g)$.

One can show that upon defining for open subsets $\mathcal{O}$ of $\mathcal{M}$ with compact closure, $\mathcal{A}(\mathcal{O})$ as the $C^{*}$-subalgebra of $\mathcal{A}[K, \kappa]$ generated by $\{W([f]) \mid f \in \mathcal{D}(\mathcal{O})\}$, $\mathcal{O} \mapsto \mathcal{A}(\mathcal{O})$ is a net of local observable algebras (see [Dim]), of which the most prominent properties are

$$
\begin{gathered}
\mathcal{A}(\mathcal{O}) \subset \mathcal{A}\left(\mathcal{O}_{1}\right) \text { for } \quad \mathcal{O} \subset \mathcal{O}_{1}, \\
{\left[\mathcal{A}(\mathcal{O}), \mathcal{A}\left(\mathcal{O}_{1}\right)\right]=\{0\}}
\end{gathered}
$$

if the regions $\mathcal{O}$ and $\mathcal{O}_{1}$ are spacelike separated,

$$
\mathcal{A}\left(\diamond\left(\mathcal{C}_{*}\right)\right) \subset \mathcal{A}(\mathcal{N})
$$

if $\mathcal{C}_{*}$ is an open subset of a Cauchy-surface and $\mathcal{N}$ an open neighbourhood, in $\mathcal{M}$, of $\mathcal{C}_{*}$.

The last relation (2.7) is clearly implied by Proposition 2.2(b). Notice also that we have

$$
\mathcal{A}\left(\diamond\left(\mathcal{C}_{*}\right)\right)=\mathcal{A}\left[K_{\mathcal{C}_{*}}, \kappa_{\mathcal{C}_{*}}\right]
$$

\section{Local Quasiequivalence}

Let $\omega$ be a state (a positive, linear, normalized functional) on the Weyl-algebra $\mathcal{A}[Z, \zeta]$ of some symplectic space $(Z, \zeta)$, and let $\left(\mathcal{H}_{\omega}, \pi_{\omega}, \Omega_{\omega}\right)$ be the GNS-representation of $\omega$. Assume now that for every $z \in Z$ the unitary one-parameter group $t \mapsto \pi_{\omega}(W(t z))$, $t \in \mathbf{R}$, is strongly continuous and that $\Omega_{\omega}$ is in the domain of definition of its generator $\Phi_{\omega}(z)$. Then the two-point function $\lambda_{\omega}$ of $\omega$ exists and is given by

$$
\lambda_{\omega}(z, \tilde{z}):=\left\langle\Phi_{\omega}(z) \Omega_{\omega}, \Phi_{\omega}(\tilde{z}) \Omega_{\omega}\right\rangle
$$

for all $z, \tilde{z} \in Z . \lambda_{\omega}$ is a bilinear form on $Z$ (with values in $\mathbf{C}$ ). 
In the case that $(Z, \zeta)=(K, \kappa)$ we call

$$
\Lambda_{\omega}(f, h):=\lambda_{\omega}([f],[h]) \quad \forall f, h \in \mathcal{D}(\mathcal{M})
$$

the spatio-temporal two-point function of $\omega$. It is easily proved that $\Lambda_{\omega}$ is a bi-solution of the KG-Eq., i.e.

$$
\Lambda_{\omega}\left(\left(\nabla^{a} \nabla_{a}+m^{2}\right) f, h\right)=0=\Lambda_{\omega}\left(f,\left(\nabla^{a} \nabla_{a}+m^{2}\right) h\right)
$$

for all $f, h \in \mathcal{D}(\mathcal{M})$, by writing $\lambda_{\omega}(.,)=.\lambda_{\omega}\left(T_{\mathcal{C}}^{-1} \circ T_{\mathcal{C}}, T_{\mathcal{C}}^{-1} \circ T_{\mathcal{C}}\right.$.) for some Cauchy-surface $\mathcal{C}$ and noting that $T_{\mathcal{C}}\left(\left(\nabla^{a} \nabla_{a}+m^{2}\right) f\right)=0$ for all $f \in \mathcal{D}(\mathcal{M})$, which follows from the definition of $T_{\mathcal{C}}$ in Proposition 2.2 and from Eq. (2.4).

Let $\omega$ be a state on the Weyl-algebra $\mathcal{A}[Z, \zeta]$ of some symplectic space $(Z, \zeta)$ and assume that the two-point function $\lambda_{\omega}$ of $\omega$ exists.

Definition 3.1. $\omega$ is a quasifree state if there exists a real scalar product $\mu_{\omega}$ on $Z$ such that:

1. $\lambda_{\omega}\left(z, \tilde{z}^{\prime}\right)=\mu_{\omega}(z, \tilde{z})+\frac{i}{2} \zeta(z, \tilde{z})$,

2. $(\zeta(z, \tilde{z}))^{2} \leq 4 \mu_{\omega}(z, z) \mu_{\omega}(\tilde{z}, \tilde{z})$

3. $\omega(W(z))=\exp \left[-\frac{1}{2} \mu_{\omega}(z, z)\right]$,

for all $z, \tilde{z} \in Z$.

If $\mathcal{H}$ is a complex Hilbert-space, we denote by $F_{s}(\mathcal{H})$ the symmetric Fock-space over the one-particle space $\mathcal{H}$, and define

$$
W^{F}(\chi):=\exp \left[i \overline{\left(a^{+}(\chi)+a(\chi)\right)}\right]
$$

for all $\chi \in \mathcal{H}$, where $a$ and $a^{+}$are the usual annihilation and creation operators, respectively. $\Omega^{F}=1 \oplus 0 \oplus 0 \ldots$ will denote the Fock-vacuum. With this notation, we quote the following characterization of quasifree states from $[\mathrm{KW}]$ :

Proposition 3.2. Let $\omega$ be a quasifree state on $\mathcal{A}[Z, \zeta]$. Then there exists, uniquely up to unitary equivalence, $a$ one-particle Hilbert-space structure for $\omega$, which consists of a pair $(\mathbf{k}, \mathcal{H})$, where $\mathcal{H}$ is a complex Hilbert-space and $\mathbf{k}: Z \rightarrow \mathcal{H}$ a real-linear injective map, with the properties

$$
\mathbf{k}(Z)+i \mathbf{k}(Z) \text { is dense in } \mathcal{H}
$$

and

$$
\langle\mathbf{k}(z), \mathbf{k}(\tilde{z})\rangle_{\mathcal{H}}=\lambda_{\omega}(z, \tilde{z})=\mu_{\omega}(z, \tilde{z})+\frac{i}{2} \zeta(z, \tilde{z})
$$

for all $z, \tilde{z} \in Z$, such that the GNS-representation $\left(\mathcal{H}_{\omega}, \pi_{\omega}, \Omega_{\omega}\right)$ is given by $\left(F_{s}(\mathcal{H}), \pi^{F}, \Omega^{F}\right)$, where

$$
\pi^{F}(W(z)):=W^{F}(\mathbf{k}(z)) \quad \forall z \in Z .
$$

( $W(z)$ are the Weyl-representers of the $\mathrm{CCR}$.)

$\omega$ is pure if and only if the range of $\mathbf{k}$ is dense in $\mathcal{H}$.

To formulate an important result which we shall use in the proof of the local quasiequivalence of quasifree Hadamard states on the Weyl-algebra of the KG-field in globally hyperbolic spacetimes we need to introduce some further concepts. Let $\omega$ be a quasifree state on the Weyl-algebra $\mathcal{A}[Z, \zeta]$, with associated real scalar product $\mu_{\omega}$ on $Z$. Denoting the completion of $Z$ with respect to the topology induced by $\mu_{\omega}$ by $\bar{Z},\left(\bar{Z}, \mu_{\omega}\right)$ becomes a real Hilbert-space. By estimate 2 of Definition 3.1, $\zeta$ 
posesses a unique extension $\bar{\zeta}$ to $\bar{Z}$ satisfying the same estimate. Then there is, by Riesz's representation theorem, a unique bounded linear operator $R$ on $\bar{Z}$ such that

$$
\bar{\zeta}(y, z)=\mu_{\omega}(y, R z)
$$

for all $y, z \in \bar{Z}$. $R$ will be called the polarizator of $\omega$. It is easily seen that $R$ is antisymmetric, i.e. $R^{*}=-R$. Another property of $R$ is derived from the following Lemma, where $\pi_{\omega}$ denotes as usual the GNS-representation of $\omega$.

Lemma 3.3. $\omega$ is primary, i.e. $\pi_{\omega}(\mathcal{A}[Z, \zeta])^{\prime \prime}$ is a factor, if and only if one of the following is fulfilled:

(a) $\bar{\zeta}$ is a symplectic form on $\bar{Z}$,

(b) $R$ is injective and its range is dense in $\bar{Z}$ so that $R^{-1}$ is a densely defined (possibly unbounded) linear operator in $\bar{Z}$.

(a) is proved in Proposition 11 and Theorem 3 of [MV].

(b) If $y \in \bar{Z}$ is such that $\mu_{\omega}(y, R z)=0$ for all $z \in \bar{Z}$, this means $\bar{\zeta}(y, z)=0$ for all $z \in \bar{Z}$ and hence $y=0$ if $\bar{\zeta}$ is a symplectic form an $\bar{Z}$. So $R$ has dense range, and injectivity follows from the antisymmetry of $R$. On the other hand, injectivity of $R$ clearly implies that $\bar{\zeta}$ is a symplectic form, i.e. (a) and (b) are equivalent.

Now consider two quasifree states $\omega_{\jmath}, j=1,2$ on $\mathcal{A}[Z, \zeta]$ and suppose that their associated real scalar products $\mu_{j}$ on $Z$ induce the same topology. It is then clear by the last Lemma that both quasifree states will be primary if and only if either of them is primary. Also, if we assume primarity and denote by $R_{j}$ the respective polarizators of $\omega_{j}$, then the following holds.

Lemma 3.4. $R_{\jmath} R_{k}^{-1}, j, k=1,2$, extend to continuous operators on $\left(\bar{Z}, \mu_{l}\right), l=1,2$.

Proof. The case $j=k$ is trivial. For the general case, observe that for all $x \in \bar{Z}$ and all $y \in \operatorname{dom}\left(R_{k}^{-1}\right)$ one has $\mu_{j}\left(x, R_{j} R_{k}^{-1} y\right)=\bar{\zeta}\left(x, R_{k}^{-1} y\right)=\mu_{k}(x, y)$ and so the lemma follows from the equivalence of the norms induced by the $\mu_{\jmath}$ and the fact that $\operatorname{dom}\left(R_{J}^{-1}\right)$ is dense in $\bar{Z}(j=1,2)$.

With the assumptions on the $\omega_{j}, j=1,2$ as before, let $Z^{\mathbf{C}}:=Z \oplus i Z$ be the standard complexification of $Z$, let $\zeta^{\mathbf{C}}$ and $\mu_{j}^{\mathbf{C}}$ be the standard complexifications ${ }^{1}$ of $\zeta$ and $\mu_{\jmath}, j=1,2$, on $Z^{\mathbf{C}}$, define $\Gamma(z \oplus i \tilde{z}):=z \oplus-i \tilde{z}$ for all $z \oplus i \tilde{z} \in Z^{\mathbf{C}}$, and set $\gamma:=i \zeta^{\mathbf{C}}$. Then $\left(Z^{\mathbf{C}}, \Gamma, \gamma\right)$ is a phase space in the sense of Araki, see [AY]. Using property 2 of Definition 3.1 , one can show that

$$
S_{j}(\mathbf{y}, \mathbf{z}):=\mu_{\jmath}^{\mathbf{C}}(\mathbf{y}, \mathbf{z})+\frac{1}{2} \gamma(\mathbf{y}, \mathbf{z}) \quad \forall \mathbf{y}, \mathbf{z} \in Z^{\mathbf{C}}
$$

are polarizations in the sense of [AY]. Then we have $(., .)_{S_{j}}=2 \mu_{\jmath}^{\mathrm{C}}$ in the notation of [AY]. Define bounded, positive C-linear operators $\$_{1}, \$_{2}$ and $\underline{S}_{2}$ and $\bar{Z}^{\mathrm{C}}$ (the closure taken with respect to the topology of any of the $\mu_{j}^{\mathrm{C}}$ as the topologies of the $\mu_{\jmath}$ are by our assumptions equivalent) by

$$
\begin{aligned}
& S_{j}(\mathbf{y}, \mathbf{z})=\left(\mathbf{y}, \$_{j} \mathbf{z}\right)_{S_{j}}, \\
& S_{2}(\mathbf{y}, \mathbf{z})=\left(\mathbf{y}, \underline{S}_{2} \mathbf{z}\right)_{S_{1}} .
\end{aligned}
$$

${ }_{1}$ Defined so that they are conjugate-linear in the first, linear in the second variable, in line with the convention used in $[\mathrm{AY}]$ 
It is easily shown that

$$
\$_{j}=\frac{1}{2}\left(\begin{array}{cc}
1 & -\frac{1}{2} R_{\jmath} \\
\frac{1}{2} R_{j} & 1
\end{array}\right)
$$

and

$$
\underline{S}_{2}=\frac{1}{2}\left(\begin{array}{cc}
R_{1} R_{2}^{-1} & -\frac{1}{2} R_{1} \\
\frac{1}{2} R_{1} & R_{1} R_{2}^{-1}
\end{array}\right),
$$

where the matrix operators act on $\mathbf{z} \in Z^{\mathrm{C}}$ as if it were a column vector. Araki and Yamagami [AY] proved that $\pi_{\omega_{1}}$ and $\pi_{\omega_{2}}$ are quasiequivalent if and only if the following two conditions are fulfilled:

(a) $(., .)_{S_{1}}$ and $(., .)_{S_{2}}$ induce the same topology on $Z^{\mathrm{C}}$, and

(b) $\$_{1}^{1 / 2}-\underline{S}_{2}^{1 / 2}$ is a Hilbert-Schmidt operator on $\left(\bar{Z}^{\mathbf{C}},(., .)_{S_{1}}\right)$.

Condition (a) is fulfilled by our assumptions. A lemma by Buchholz (cf. Appendix $\mathrm{B}$ in [Buc]) states that (b) is fulfilled if $\$_{1}-\underline{S}_{2}$ is a trace-class operator on $\left(\bar{Z}^{\mathrm{C}},(., .)_{S_{1}}\right)$. This is, using (3.4) and (3.5), the case if $\mathbf{1}-R_{1} R_{2}^{-1}$ is a trace-class operator on $\left(\bar{Z}, \mu_{1}\right)$. So we obtain the following corollary to the result by Araki and Yamagami:

Corollary 3.5. The GNS-representations $\pi_{\omega_{j}}$ of two primary quasifree states $\omega_{j}$, $j=1,2$, whose associated real scalar products $\mu_{j}$ induce the same topology on $Z$ are quasiequivalent if $\mathbf{1}-R_{1} R_{2}^{-1}$ is a trace-class operator on $\left(\bar{Z}, \mu_{1}\right)$.

Another notion from spacetime geometry, due to Kay and Wald [KW], will be needed.

Definition 3.6. Let $\mathcal{C}$ be a Cauchy-surface for $(\mathcal{M}, g)$. An open neighbourhood $\mathcal{N}$ of $\mathcal{C}$ is a causal normal neighbourhood of $\mathcal{C}$ if $\mathcal{C}$ is a Cauchy-surface for $\mathcal{N}$ and if for $p, q \in \mathcal{N}$ with $p \in J^{+}(q)$, there exists a convex normal neighbourhood $\mathcal{O}$ in $\mathcal{M}$ such that $J^{-}(p) \cap J^{+}(q) \subset \mathcal{O}$.

The existence of such causal normal neighbourhoods is guaranteed by Lemma 2.2 in $[\mathrm{KW}]$. Now let $\omega_{\jmath}, j=1,2$ be two quasifree states on the Weyl-algebra $\mathcal{A}[K, \kappa]$ of the KG-field in the globally hyperbolic spacetime $(\mathcal{M}, g)$. Their associated spatiotemporal two-point functions will be denoted by $\Lambda_{j}$. We say that the $\Lambda_{j}$ are locally distributions if for each $p \in \mathcal{M}$ there is a neighbourhood $\mathcal{O}$ of $p$ such that the $\Lambda_{\text {J }}$ can be identified with elements of $\mathcal{D}_{\mathrm{C}}^{\prime}(\mathcal{O} \times \mathcal{O})$. We say that $\Lambda_{1}-\Lambda_{2}$ is a smooth bisolution near Cauchy-surfaces if every Cauchy-surface $\mathcal{C}$ possesses a causal normal neighbourhood $\mathcal{N}$ such that

$$
\Lambda_{1}(f, h)-\Lambda_{2}(f, h)=\int f(p) B(p, q) h(q) d \eta(p) d \eta(q)
$$

for all $f, h \in \mathcal{D}(\mathcal{N})$, where $B \in C^{\infty}(\mathcal{N} \times \mathcal{N})$ is a bi-solution, i.e.

$$
\left(\nabla^{a} \nabla_{a}+m^{2}\right)_{p} B(p, q)=0=\left(\nabla^{a} \nabla_{a}-m^{2}\right)_{q} B(p, q)
$$

for all $p, q \in \mathcal{N}$.

The following lemma will be of some importance.

Lemma 3.7. Let $\mathcal{N}$ be a causal normal neighbourhood of some Cauchy-surface in $(\mathcal{M}, g), B$ a smooth bi-solution of the KG-Eq. on $\mathcal{N} \times \mathcal{N}$ and $G$ an open subset of $\mathcal{N}$ 
with compact closure in $\mathcal{N}$. Then there is some $Q \in C_{0}^{\infty}(\mathcal{N} \times \mathcal{N})$ such that

$$
\int f(p) B(p, q) h(q) d \eta(p) d \eta(q)=\int(E f)(p) Q(p, q)(E h)(q) d \eta(p) d \eta(q)
$$

for all $f, h \in \mathcal{D}(G)$. E is the propagator of the $K G-E q$.

We shall prove this lemma in Appendix A.

Now we state and prove our main technical result.

Proposition 3.8. Assume that the spatio-temporal two-point functions $\Lambda_{j}(j=1,2)$ of two quasifree states on $\mathcal{A}[K, \kappa]$ are locally distributions and that $\Lambda_{1}-\Lambda_{2}$ is a smooth bi-solution near Cauchy-surfaces. Let $\mathcal{N}$ be a causal normal neighbourhood of some Cauchy-surface and $\mathcal{O}$ an open subset of $\mathcal{M}$ with compact closure. Then there are sequences $\phi_{\jmath}, \psi_{\jmath} \in \mathcal{D}(\mathcal{N}), j \in \mathbf{N}$, with the properties:

$$
\Lambda_{1}(f, h)-\Lambda_{2}(f, h)=\sum_{j \in \mathbf{N}} \kappa\left([f],\left[\phi_{j}\right]\right) \kappa\left([h],\left[\psi_{j}\right]\right)
$$

for all $f, h \in \mathcal{D}(\diamond(\mathcal{O}))$.

$$
\sum_{j \in \mathbf{N}} \Lambda_{k}\left(\phi_{j}, \phi_{j}\right)^{1 / 2} \Lambda_{k}\left(\psi_{j} \psi_{j}\right)^{1 / 2}<\infty, \quad k=1,2,
$$

implying that there are $\alpha, \beta>0$ such that

$$
\alpha \Lambda_{1}(f, f) \leq \Lambda_{2}(f, f) \leq \beta \Lambda_{1}(f, f)
$$

for all $f \in \mathcal{D}(\diamond(\mathcal{O}))$.

Proof. We shall first show how (a) and (b) together imply (c): One obtains

$$
\begin{aligned}
\left|\Lambda_{1}(f, f)-\Lambda_{2}(f, f)\right| & \left.\leq \sum_{j \in \mathbf{N}}\left|\kappa\left([f],\left[\phi_{\jmath}\right]\right)\right| \mid \kappa[f],\left[\psi_{j}\right]\right) \mid \\
& \leq \sum_{j \in \mathbf{N}} 4 \Lambda_{k}(f, f) \Lambda_{k}\left(\phi_{j}, \phi_{j}\right)^{1 / 2} \Lambda_{k}\left(\psi_{\jmath}, \psi_{\jmath}\right)^{1 / 2}
\end{aligned}
$$

for all $f \in \mathcal{D}(\diamond(\mathcal{O})), k=1,2$, and hence

$$
\Lambda_{1}(f, f) \leq \Lambda_{2}(f, f)\left(1+\sum_{j \in \mathbf{N}} 4 \Lambda_{2}\left(\phi_{j}, \phi_{j}\right)^{1 / 2} \Lambda_{2}\left(\psi_{\jmath}, \psi_{\jmath}\right)^{1 / 2}\right)
$$

for all $f \in \mathcal{D}(\diamond(\mathcal{O}))$. The same estimate holds when interchanging the indices $1 \leftrightarrow 2$. Now we show how to construct the sequences $\phi_{\jmath}, \psi_{\jmath}, j \in \mathbf{N}$, with the properties (a) and (b). Let $\mathcal{C}$ be the Cauchy-surface of which $\mathcal{N}$ is a causal normal neighbourhood. Then there is some $\mathcal{C}_{*} \subset \mathcal{C}$ with compact closure such that $\mathcal{O} \subset \diamond\left(\mathcal{C}_{*}\right)$. So $f \in \mathcal{D}(\diamond(\mathcal{O}))$ implies that $[f] \in K_{\mathcal{C}_{*}}$. Note that we have

$$
\Lambda_{1}(f, h)-\Lambda_{2}(f, h)=\lambda_{\omega_{1}}([f],[h])-\lambda_{\omega_{2}}([f],[h])
$$

for all $f, h \in \mathcal{D}\left(\diamond\left(\mathcal{C}_{*}\right)\right)$. Now let $G$ be a neighbourhood, in $\mathcal{N}$, of $\mathcal{C}_{*}$ such that $G$ has compact closure in $\mathcal{N}$. According to Proposition 2.2(b) we find for each $[f] \in K_{\mathcal{C}_{*}}$ some $\tilde{f} \in \mathcal{D}(G)$ such that $[f]=[\tilde{f}]$. Using this and (3.9), it suffices for the proof of the proposition to construct sequences $\phi_{j}, \psi_{j} \in \mathcal{D}(\mathcal{N})$ with the property (b), and such that

$$
\left|\Lambda_{1}(f, h)-\Lambda_{2}(f, h)\right|=\sum_{j \in \mathbf{N}} \kappa\left([f],\left[\phi_{j}\right]\right) \kappa\left([h],\left[\psi_{j}\right]\right)
$$


for all $f, h \in \mathcal{D}(G)$. This will be done as follows. By Lemma 3.7 there is some $Q \in C_{0}^{\infty}(\mathcal{N} \times \mathcal{N})$ such that for all $f, h \in \mathcal{D}(G)$,

$$
\begin{aligned}
\Lambda_{1}(f, h)-\Lambda_{2}(f, h) & =\int f(p) B(p, q) h(q) d \eta(p) d \eta(q) \\
& =\int E f(p) Q(p, q) \operatorname{Eh}(q) d \eta(p) d \eta(q)
\end{aligned}
$$

and we may assume that $\operatorname{supp}(Q) \subset \mathcal{G} \times \mathcal{G}$, where $\mathcal{G}$ is some compact set contained in $\mathcal{N}$. By $\Psi_{p}$ we denote a chart around $p \in \mathcal{N}$ with the following properties: (i) $\operatorname{dom}\left(\Psi_{p}\right)$ is contained in a neighbourhood $\mathcal{O}$ of $p$ for which the $\Lambda_{k}, k=1,2$, can be identified with elements of $\mathcal{D}_{\mathbf{C}}^{\prime}(\mathcal{O} \times \mathcal{O})$, and (ii) the closure of $I^{4}$, where $I:=(-\pi, \pi)$, is contained in $\operatorname{Ran}\left(\Psi_{p}\right)$. Choose some $\varepsilon \in(0,1)$, set $I_{\varepsilon}:=(-\pi+\varepsilon, \pi-\varepsilon)$ and $U_{p}(\varepsilon):=\Psi_{p}^{-1}\left(I_{\varepsilon}^{4}\right)$. The family of neighbourhoods $U_{p}(\varepsilon), p \in \mathcal{N}$, forms an open covering of $\mathcal{N}$. Then one can choose a locally finite partition of unity (of $\mathcal{N}$ ) $\left\{\varrho_{\sigma}\right\}_{\sigma \in S}$ subordinate to the cover $U_{p}(\varepsilon), p \in \mathcal{N}$. Then $\mathcal{G}$ has non-void intersection with the supports of only finitely many elements of the partition of unity, which will be denoted by $\varrho_{1}, \ldots, \varrho_{k}$. Therefore we have:

$$
\Lambda_{1}(f, h)-\Lambda_{2}(f, h)=\sum_{r, s=1}^{k} \int \varrho_{r}(p) \varrho_{s}(q) Q(p, q) E f(p) E h(q) d \eta(p) d \eta(q)
$$

for all $f, h \in \mathcal{D}(G)$. By construction, the smooth function $Q_{r s}:=\left(\varrho_{r} \otimes \varrho_{s}\right) Q$, $r, s=1, \ldots, k$, has compact support in $U_{p(r)}(\varepsilon) \times U_{q(s)}(\varepsilon)$ for some $p(r), q(s) \in \mathcal{N}$, and hence $Q_{r s} \circ\left(\Psi_{p(r)} \times \Psi_{q(s)}\right)^{-1}$ is a smooth function with support in $I_{\varepsilon}^{4} \times I_{\varepsilon}^{4}$. From what we show in Appendix $B$ it then follows that there are sequences $\phi_{\nu}^{(r)} \in \mathcal{D}\left(U_{p(r)}(\varepsilon)\right), \psi_{\nu}^{(s)} \in \mathcal{D}\left(U_{q(s)}(\varepsilon)\right), \nu \in \mathbf{N}, r, s=1, \ldots, k$, such that

$$
\begin{aligned}
\int & Q_{r s}(p, q) E f(p) E h(q) d \eta(p) d \eta(q) \\
= & \sum_{\nu \in \mathbf{N}} \int \phi_{\nu}^{(r)}(p) \psi_{\nu}^{(s)}(q) E f(p) E h(q) d \eta(p) d \eta(q) \\
= & \sum_{\nu \in \mathbf{N}} \kappa\left([f],\left[\phi_{\nu}^{(r)}\right]\right) \kappa\left([h],\left[\psi_{\nu}^{(s)}\right]\right)
\end{aligned}
$$

for all $f, h \in \mathcal{D}(G), r, s=1, \ldots, k$, and

$$
\sum_{\nu \in \mathbf{N}} \Lambda_{l}\left(\phi_{\nu}^{(r)}, \phi_{\nu}^{(r)}\right)^{1 / 2} \Lambda_{l}\left(\psi_{\nu}^{(s)}, \psi_{\nu}^{(s)}\right)^{1 / 2}<\infty
$$

for $l=1,2, r, s=1, \ldots, k$, since the $\Lambda_{l}$ are by construction identifyable with elements of $\mathcal{D}_{\mathbf{C}}^{\prime}\left(U_{p(r)}(0) \times U_{p(r)}(0)\right)$ and of $\mathcal{D}_{\mathbf{C}}^{\prime}\left(U_{q(s)}(0) \times U_{q(s)}(0)\right)$. Now choose a denumeration $j \mapsto\left(r_{j}, s_{j}, \nu_{j}\right), j \in \mathbf{N}$ of the index triples $r, s \stackrel{q}{=} 1, \ldots, k, \nu \in \mathbf{N}$, and set $\phi_{j}:=\phi_{\nu_{j}}^{\left(r_{j}\right)}, \psi_{j}:=\psi_{\nu_{j}}^{\left(s_{\jmath}\right)}$. Then we find with (3.11-13)

$$
\Lambda_{1}(f, h)-\Lambda_{2}(f, h)=\sum_{j \in \mathbf{N}} \kappa\left([f],\left[\phi_{j}\right]\right) \kappa\left([h],\left[\psi_{j}\right]\right)
$$


for all $f, h \in \mathcal{D}(G)$ and

$$
\sum_{j \in \mathbf{N}} \Lambda_{l}\left(\phi_{j}, \phi_{j}\right)^{1 / 2} \Lambda_{l}\left(\psi_{j}, \psi_{j}\right)^{1 / 2}<\infty, \quad l=1,2 .
$$

This completes the proof.

Until now we have not mentioned the term "Hadamard state." We will define Hadamard states on the Weyl-algebra of the $\mathrm{KG}$-field in $(\mathcal{M}, g)$ in Appendix $\mathrm{C}$. Hadamard states are states for which the spatio-temporal two-point function takes a special "Hadamard" form. One can show that spatio-temporal two-point functions of Hadamard form fulfill the assumptions of Proposition 3.8 (cf. Appendix C), i.e. they are locally distributions and their differences are smooth bisolutions near Cauchysurfaces. Spatio-temporal two-point functions of Hadamard form possess another remarkable property, derived by [FSW; KW], which we refer to as the causal propagation property of the Hadamard form: This means that if a spatio-temporal two-point function is of Hadamard form near some arbitrary Cauchy-surface in $\mathcal{M}$, then it is of Hadamard form near all Cauchy-surfaces. (See Appendix C for a precise form of this statement.)

Now we are ready to present the result.

Theorem 3.9. Let $\omega_{1}$ and $\omega_{2}$ be two quasifree Hadamard states on the Weyl-algebra $\mathcal{A}[K, \kappa]$ of the $\mathrm{KG}$-field in the globally hyperbolic spacetime $(\mathcal{M}, g)$, and let $\pi_{1}$ and $\pi_{2}$ be their associated GNS-representations. Then $\pi_{1} \mid \mathcal{A}(\mathcal{O})$ and $\pi_{2} \mid \mathcal{A}(\mathcal{O})$ are quasiequivalent for every open subset $\mathcal{O}$ of $\mathcal{M}$ with compact closure.

Proof. The proof has two main parts, of which the first involves more "geometrical," the second more "functional analytical" elements.

1. The first part of the proof consists in explaining how, using the method of "deforming a globally hyperbolic spacetime in the past to an ultrastatic one while preserving global hyperbolicity" (due to [FNW]) and the causal propagation property of the Hadamard form, the problem can be reduced to a more specialized situation.

Let $\omega_{1}$ and $\omega_{2}$ be two quasifree, Hadamard states on the Weyl-algebra $\mathcal{A}[K, \kappa]$ of the KG-field in a globally hyperbolic spacetime $(\mathcal{M}, g)$ and $\pi_{j}, j=1,2$, their associated GNS-representations. First notice that, if $\pi_{1} \mid \mathcal{A}(\mathcal{O})$ and $\pi_{2} \mid \mathcal{A}(\mathcal{O})$ are quasiequivalent, then $\pi_{1} \mid \mathcal{A}\left(\mathcal{O}_{*}\right)$ and $\pi_{2} \mid \mathcal{A}\left(\mathcal{O}_{*}\right)$ are quasiequivalent for $\mathcal{O}_{*} \subset \mathcal{O}$. Then notice that, given a Cauchy-surface $\mathcal{C}$ for $(\mathcal{M}, g)$, and an open subset $\mathcal{O}$ of $\mathcal{M}$ with compact closure, we find some subset $\mathcal{C}_{*}$ of $\mathcal{C}$ with compact closure such that $\mathcal{O} \subset \nabla\left(\mathcal{C}_{*}\right)$, and given any neighbourhood $\mathcal{N}$ of $\mathcal{C}$, we have $\mathcal{A}(\mathcal{O}) \subset \mathcal{A}\left(\diamond\left(\mathcal{C}_{*}\right) \cap \mathcal{N}\right)$, c.f. (2.7). So for the proof of local quasiequivalence we may concentrate on regions of the type $\nabla\left(\mathcal{C}_{*}\right) \cap \mathcal{N}$. Given the Cauchy-surface $\mathcal{C}$, one can find a neighbourhood $\mathcal{N}$ of $\mathcal{C}$ and a globally hyperbolic spacetime $(\widehat{\mathcal{M}}, \hat{g})$ with the following properties:

(i) There is a time-orientation preserving isometry $\phi:(\mathcal{N}, g \mid \mathcal{N}) \rightarrow(\widehat{\mathcal{N}}, \hat{g} \mid \widehat{\mathcal{N}})$, where $\widehat{\mathcal{N}}$ is a neighbourhood of a Cauchy-surface $\widehat{\mathcal{C}}$ in $(\widehat{\mathcal{M}}, \hat{g}) . \phi$ induces also an isometry between $\mathcal{C}$ and $\widehat{\mathcal{C}}$.

(ii) To the past of some Cauchy-surface $\widehat{\mathcal{C}}^{\prime}$ lying to the past of $\widehat{\mathcal{C}},(\widehat{\mathcal{M}}, \hat{g})$ equals the $(-\infty, 0) \times \widehat{\mathcal{C}}$-part of an ultrastatic, globally hyperbolic spacetime foliated by some complete, three-dimensional Riemannian manifold $(\widehat{\mathcal{C}}, \hat{\gamma})$.

The construction of such an $(\widehat{\mathcal{M}}, \hat{g})$ is demonstrated in Appendix $\mathrm{C}$ of [FNW]. Notice that we may assume that the neighbourhood $\mathcal{N}$ of $\mathcal{C}$ is a causal normal neigh- 
bourhood of $\mathcal{C}$, since every neighbourhood of $\mathcal{C}$ contains a causal normal neighbourhood of $\mathcal{C}$, as can be seen from the proof of Lemma 2.2 in [KW]. The isometry $\phi$ of (i) induces canonically a $C^{*}$-algebraic isomorphism $\alpha: \mathcal{A}[K, \kappa] \rightarrow \widehat{\mathcal{A}}[\widehat{K}, \hat{\kappa}]$, where $\widehat{\mathcal{A}}[\widehat{K}, \hat{\kappa}]$ is the Weyl-algebra of the KG-field in $(\widehat{\mathcal{M}}, \hat{g}) . \alpha$ is also an isomorphism between the corresponding nets of $C^{*}$-algebras, i.e. $\alpha(\mathcal{A}(\mathcal{O}))=\widehat{\mathcal{A}}(\phi(\mathcal{O}))$ (see [Dim]). Since the Hadamard form of spatio-temporal two-point functions is preserved under time-orientation preserving spacetime-isometries, and since we have the causal propagation property of the Hadamard form, it follows that the quasifree Hadamard states $\omega_{j}$ on $\mathcal{A}[K, \kappa]$ induce quasifree Hadamard states $\hat{\omega}_{j}:=\omega_{j} \circ \alpha^{-1}$ on $\widehat{\mathcal{A}}[\widehat{K}, \hat{\kappa}]$. Denoting their GNS-representations by $\hat{\pi}_{j}$, it is now easily seen that $\pi_{1}$ and $\pi_{2}$ are locally quasiequivalent if the $\hat{\pi}_{j} \mid \widehat{\mathcal{A}}(\widehat{\mathcal{O}})$ are quasiequivalent for arbitrary open regions $\widehat{\mathcal{O}}$ with compact closure in $\widehat{\mathcal{N}}$. Now let $\widehat{\mathcal{C}_{t}}$, for some $t<0$, be a Cauchy-surface in the natural foliation of the ultrastatic part of $(\widehat{\mathcal{M}}, \hat{g})$, and $\widehat{\mathcal{N}}$ and arbitrary neighbourhood of $\widehat{\mathcal{C}_{t}}$. Then, by the same arguments as given above, $\widehat{\mathcal{A}}(\widehat{\mathcal{O}}) \subset \widehat{\mathcal{A}}\left(\widehat{\nabla}\left(\widehat{\mathcal{C}}_{\#}\right) \cap \widehat{\mathcal{N}}^{\prime}\right)$ for some open subset $\mathcal{C}_{\#}$ of $\widehat{\mathcal{C}_{t}}$ with compact closure whose boundary $\partial \mathcal{C}_{\#}$ is contained in the union of finitely many smooth, closed (even compact), two-dimensional submanifolds of $\widehat{\mathcal{C}_{t}}$. (To see this, note that any compact subset of $\widehat{\mathcal{C}_{t}}$ may be covered by finitely many "normal coordinate balls" whose boundaries are compact hypersurfaces in $\widehat{\mathcal{C}_{t}}$.)

Therefore, it suffices to show that the $\hat{\pi}_{j} \mid \widehat{\mathcal{A}}\left(\hat{\nabla}\left(\mathcal{C}_{\#}\right) \cap \widehat{\mathcal{N}}^{\prime}\right), j=1,2$, are quasiequivalent for the GNS-representations $\hat{\pi}_{j}$ of any two quasifree Hadamard states $\hat{\omega}_{j}, j=1,2$, on $\widehat{\mathcal{A}}[\widehat{K}, \hat{\kappa}]$, where $\mathcal{C}_{\#}$ is an arbitrary open subset of $\widehat{\mathcal{C}}_{t}$ with compact closure such that $\partial \mathcal{C}_{\#}$ is contained in the union of finitely many smooth, closed, two-dimensional submanifolds of $\widehat{\mathcal{C}_{t}}$, and $\widehat{\mathcal{N}}^{\prime}$ is a causal normal neighbourhood of $\widehat{\mathcal{C}_{t}}$. This is the more specialized form of the problem we desired to arrive at.

2. The second part of the proof can be subdivided into

(a) proving that $\hat{\pi}_{j}\left(\hat{\mathcal{A}}\left(\hat{\diamond}\left(\mathcal{C}_{\#}\right)\right)\right)^{\prime \prime}, j=1,2$, are factors, and

(b) showing how this factoriality, together with our Proposition 3.8, entails the quasiequivalence of the $\hat{\pi}_{j} \mid \widehat{\mathcal{A}}\left(\hat{\nabla}\left(\mathcal{C}_{\#}\right)\right), j=1,2$.

The task (a) will be carried out in the next chapter.

(b) The GNS-representations $\tilde{\pi}_{1}$ and $\tilde{\pi}_{2}$ of the states $\tilde{\omega}_{j}:=\tilde{\omega}_{j} \mid \widehat{\mathcal{A}}\left(\hat{\nabla}\left(\mathcal{C}_{\#}\right)\right), j=1,2$ are subrepresentations of the representations $\hat{\pi}_{j} \mid \widehat{\mathcal{A}}\left(\hat{\nabla}\left(\mathcal{C}_{\#}\right)\right), j=1,2$, and hence it follows, by a standard result (cf. Appendix E.1), from the factoriality property (a) that $\tilde{\pi}_{j}$ is quasiequivalent to $\hat{\pi}_{j} \mid \widehat{\mathcal{A}}\left(\hat{\nabla}\left(\mathcal{C}_{\#}\right)\right), j=1,2$. So the $\hat{\pi}_{j} \mid \widehat{\mathcal{A}}\left(\hat{\nabla}\left(\mathcal{C}_{\#}\right)\right), j=1,2$, are quasiequivalent if $\tilde{\pi}_{1}$ and $\tilde{\pi}_{2}$ are quasiequivalent (see Appendix E.1 for details of this argument), and we show that the latter is the case. The factoriality property (a) entails that the $\tilde{\omega}_{j}$ are primary. Let $\Lambda_{j}, \mu_{j}, j=1,2$, be the spatio-temporal two-point distributions, and real scalar products on $\widehat{K}$, respectively, of the states $\hat{\omega}_{j}$ on $\widehat{\mathcal{A}}[\widehat{K}, \hat{\kappa}]$; and denote by $\tilde{\Lambda}_{j}, \tilde{\mu}_{j}$ the corresponding objects of the states $\tilde{\omega}_{j}$ on $\widehat{\mathcal{A}}\left[\widehat{K}_{\mathcal{C}_{\#}}, \hat{\kappa}_{\mathcal{C}_{\#}}\right]$. Let $\tilde{R}_{j}, j=1,2$, be the polarizators of the $\tilde{\omega}_{j}$. By Proposition 3.8(c) the $\tilde{\mu}_{j}, j=1,2$, induce the same topology on $\widehat{K}_{\mathcal{C}_{\sharp}}$. Therefore we see from Corollary 3.5 that the quasiequivalence of the $\tilde{\pi}_{j}, j=1,2$, is proven if $\mathbf{1}-\tilde{R}_{1} \tilde{R}_{2}^{-1}$ is a traceclass operator on $\left(\widehat{\widehat{K}}_{\mathcal{C}_{\#}}, \tilde{\mu}_{1}\right)$. To show that this is the case, recall that by Proposition 3.8 there are 
sequences $\phi_{\nu}, \psi_{\nu} \in \mathcal{D}\left(\widehat{\mathcal{N}}^{\prime}\right), \nu \in \mathbf{N}$, such that

$$
\begin{aligned}
\tilde{\mu}_{1}([f],[h])-\tilde{\mu}_{2}([f],[h]) & =\Lambda_{1}(f, h)-\Lambda_{2}(f, h) \\
& =\sum_{\nu \in \mathbf{N}} \hat{\kappa}\left([f],\left[\phi_{\nu}\right]\right) \hat{\kappa}\left([h],\left[\psi_{\nu}\right]\right)
\end{aligned}
$$

for all $[f],[h] \in \widehat{K}_{\mathcal{C}_{\#}}$ and

$$
\sum_{\nu \in \mathbf{N}} \mu_{1}\left(\left[\phi_{\nu}\right],\left[\phi_{\nu}\right]\right)^{1 / 2} \mu_{1}\left(\left[\psi_{\nu}\right],\left[\psi_{\nu}\right]\right)^{1 / 2}<\infty
$$

Now let $R_{1}$ be the polarizator of $\hat{\omega}_{1}$ and $P$ the projector in $\left(\overline{\widehat{K}}, \mu_{1}\right)$ onto the closed subspace $\widehat{\widehat{K}}_{\mathcal{C}_{\#}}$, and define

$$
x_{\nu}:=P R_{1}\left[\phi_{\nu}\right], \quad y_{\nu}:=P R_{1}\left[\psi_{\nu}\right], \quad \nu \in \mathbf{N} .
$$

Thus we obtain

$$
\begin{aligned}
& \hat{\kappa}\left([f],\left[\phi_{\nu}\right]\right)=\mu_{1}\left([f], R_{1}\left[\phi_{\nu}\right]\right)=\tilde{\mu}_{1}\left([f], x_{\nu}\right), \\
& \hat{\kappa}\left([h],\left[\psi_{\nu}\right]\right)=\mu_{1}\left([h], R_{1}\left[\psi_{\nu}\right]\right)=\tilde{\mu}_{1}\left([h], y_{\nu}\right),
\end{aligned}
$$

for all $[f],[h] \in \overline{\widehat{K}}_{\mathcal{C}_{\#}}$ and for all $\nu \in \mathbf{N}$, and since $P$ and $R_{1}$ are bounded operators we infer from (3.17) that

$$
\sum_{\nu \in \mathbf{N}} \tilde{\mu}_{1}\left(x_{\nu}, x_{\nu}\right)^{1 / 2} \tilde{\mu}_{1}\left(y_{\nu}, y_{\nu}\right)^{1 / 2}<\infty
$$

On the other hand, Eqs. (3.16-20) imply that

$$
\begin{aligned}
\tilde{\mu}_{1}\left([f],\left(\mathbf{1}-\tilde{R}_{1} \tilde{R}_{2}^{-1}\right)[h]\right) & =\tilde{\mu}_{1}([f],[h])-\tilde{\mu}_{2}([f],[h]) \\
& =\sum_{\nu \in \mathbf{N}} \tilde{\mu}_{1}\left([f], x_{\nu}\right) \tilde{\mu}_{1}\left([h], y_{\nu}\right)
\end{aligned}
$$

for all $[f],[h] \in \widehat{K}_{\mathcal{C}_{\#}}$. Since $\widehat{K}_{\mathcal{C}_{\#}}$ is dense in $\widehat{\widehat{K}}_{\mathcal{C}_{\#}}$, Eqs. (3.20-21) together with the known characterizations of trace-class operators (cf. Theorem 7.12 in [Wei]) imply that $\mathbf{1}-\tilde{R}_{1} \tilde{R}_{2}^{-1}$ is a trace-class operator in $\left(\overline{\widehat{K}}_{\mathcal{C}_{\#}}, \tilde{\mu}_{1}\right)$, and hence we have proved the theorem.

\section{Local Primarity and Definiteness}

In this section we shall derive the local primarity and local definiteness of quasifree Hadamard states on the Weyl-algebra of the KG-field in ultrastatic, globally hyperbolic spacetimes. More precisely, what we are looking at is the following situation: Let $(\mathcal{M}, g)$ be the globally hyperbolic, ultrastatic space-time foliated by the complete, three-dimensional $C^{\infty}$-Riemannian manifold $(M, \gamma)$. Let $M_{t}, t \in \mathbf{R}$, be the natural foliation of $(\mathcal{M}, g)$ in Cauchy-surfaces, and for some $t \in \mathbf{R}, \mathcal{C}_{*}$ an open subset of $M_{t}$ with compact closure such that $\partial \mathcal{C}_{*}$, the boundary of $\mathcal{C}_{*}$, is contained in the union of finitely many (possibly intersecting) smooth, closed, two-dimensional submanifolds of $M_{t}$. Then we obtain the following result. 
Theorem 4.1. Let $\omega$ be a quasifree Hadamard state on the Weyl-algebra $\mathcal{A}[K, \kappa]$ of the KG-field in $(\mathcal{M}, g)$, and $\pi_{\omega}$ its GNS-representation. Then $\mathcal{R}_{\omega}\left(\diamond\left(\mathcal{C}_{*}\right)\right)=$ $\pi_{\omega}\left(\mathcal{A}\left(\diamond\left(\mathcal{C}_{*}\right)\right)\right)^{\prime \prime}$ is a factor.

Proof. Let $(\mathbf{k}, \mathcal{H})$ be the one-particle Hilbert-space structure of $\omega$. Define $\mathcal{L}:=\mathbf{k}\left(K_{\mathcal{C}_{*}}\right)$ and $\mathcal{L}^{\mathbf{v}}:=\{\chi \in \mathcal{H} \mid \operatorname{Im}\langle\chi, \theta\rangle=0 \forall \theta \in \mathcal{L}\}$ (notice that $\mathcal{L}$ is a real-linear, but in general not a complex-linear subspace of $\mathcal{H}$ ). Observe that by (2.8) and by Proposition 3.2 we have $\mathcal{R}_{\omega}\left(\diamond\left(\mathcal{C}_{*}\right)\right)=W^{F}(\mathcal{L})^{\prime \prime}$. According to results by Araki [Ara], or by Leyland, Roberts and Testard [LRT], $W^{F}(\mathcal{L})^{\prime \prime}$ is a factor if and only if $\overline{\mathcal{L}} \cap \mathcal{L}^{\mathbf{v}}=\{0\}$. To show that this is the case, we note that there is a pure, quasifree Hadamard state $\hat{\omega}$ on $\mathcal{A}[K, \kappa]$ with the property that $K_{\mathcal{C}_{*}}+K_{\left(M_{t} \backslash \overline{\mathcal{C}_{*}}\right.}$ is dense in $K$ with respect to the norm given by $\mu_{\hat{\omega}}$ on $K$. The existence of such an $\hat{\omega}$, the "canonical vacuum," will be established below. Denote by $(\hat{\mathbf{k}}, \widehat{\mathcal{H}})$ the one-particle Hilbert-space structure of $\hat{\omega}$ and set $\widehat{\mathcal{L}}:=\hat{\mathbf{k}}\left(K_{\mathcal{C}_{*}}\right)$. $\widehat{\mathcal{L}}^{\mathbf{v}}$ will be defined in the same manner as before. Notice that we have $\hat{\mathbf{k}}\left(K_{\left(M_{t} \backslash \overline{\mathcal{C}_{*}}\right)}\right) \subset \widehat{\mathcal{L}}^{\mathbf{v}}:$ For if $[f] \in K_{\left(M_{t} \backslash \overline{\mathcal{C}_{*}}\right)}$, we have for all $[h] \in K_{\mathcal{C}_{*}}$,

$$
\operatorname{Im}\langle\hat{\mathbf{k}}[f], \hat{\mathbf{k}}[h]\rangle=\int f E h d \eta=0,
$$

since $\operatorname{supp}(f) \cap \operatorname{supp}(E h) \subset \diamond\left(\mathcal{C}_{*}\right) \cap J\left(M_{t} \backslash \overline{\mathcal{C}_{*}}\right)=\emptyset$. Now suppose that $\hat{\chi} \in \overline{\widehat{\mathcal{L}}} \cap \widehat{\mathcal{L}}^{\mathrm{v}}$. Then $\operatorname{Im}\langle\hat{\chi}, \hat{\mathbf{k}}[f]\rangle=0$ for all $[f] \in K_{\mathcal{C}_{*}}$ and all $[f] \in K_{\left(M_{t} \backslash \overline{\mathcal{C}_{*}}\right)}$. By the mentioned denseness property this implies $\operatorname{Im}\langle\hat{\chi}, \hat{\mathbf{k}}(K)\rangle=\{0\}$. Since $\hat{\omega}$ is pure, $\hat{\mathbf{k}}(K)$ is dense in $\widehat{\mathcal{H}}$ by Proposition 3.2 , implying $\hat{\chi}=0$. So the theorem is proven for $\hat{\omega}$, and we shall carry over this result to our arbitrarily chosen quasifree, Hadamard state $\omega$. Assume that $\chi \in \overline{\mathcal{L}} \cap \widehat{\mathcal{L}}^{\mathbf{v}}$. Then there is a sequence $\left[f_{n}\right] \in K_{\mathcal{C}_{*}}, n \in \mathbf{N}$, with $\mathbf{k}\left[f_{n}\right] \rightarrow \chi$ in $\mathcal{H}$. By Proposition 3.8(c) we find that the norms given by $\mu_{\omega}$ and $\mu_{\hat{\omega}}$ on $K_{\mathcal{C}_{*}}$ are equivalent, and so we have $\hat{\mathbf{k}}\left[f_{n}\right] \rightarrow \hat{\chi}$ in $\widehat{\mathcal{H}}$ for some $\hat{\chi} \in \overline{\widehat{\mathcal{L}}}$. On the other hand, we have for all $[h] \in K_{\mathcal{C}_{*}}$,

$$
\begin{aligned}
0 & =\operatorname{Im}\langle\chi, \mathbf{k}[h]\rangle \\
& =\lim _{n \rightarrow \infty} \operatorname{Im}\left\langle\mathbf{k}\left[f_{n}\right], \mathbf{k}[h]\right\rangle \\
& =\lim _{n \rightarrow \infty} \frac{1}{2} \kappa\left(\left[f_{n}\right],[h]\right) \\
& =\lim _{n \rightarrow \infty} \operatorname{Im}\left\langle\hat{\mathbf{k}}\left[f_{n}\right], \hat{\mathbf{k}}[h]\right\rangle \\
& =\operatorname{Im}\langle\hat{\chi}, \hat{\mathbf{k}}[h]\rangle .
\end{aligned}
$$

This implies that $\hat{\chi} \in \overline{\widehat{\mathcal{L}}} \cap \widehat{\mathcal{L}}^{\mathbf{v}}=\{0\}$, so $\hat{\mathbf{k}}\left[f_{n}\right] \rightarrow 0$. The equivalence of the norms of $\mu_{\omega}$ and $\mu_{\hat{\omega}}$ then implies that $\chi=\lim _{n \rightarrow \infty} \mathbf{k}\left[f_{n}\right]=0$, which was what we had to show.

In the same situation as described before, we obtain local definiteness:

Theorem 4.2. Let $\omega$ be a quasifree Hadamard state on the Weyl-algebra $\mathcal{A}[K, \kappa]$ of the $\mathrm{KG}$-field in $(\mathcal{M}, g)$, and $p \in \mathcal{M}$. Then

$$
\mathcal{R}_{\omega}(p):=\bigcap_{\mathcal{O} \ni p} \mathcal{R}_{\omega}(\mathcal{O})=\mathbf{C 1}
$$

Proof. Clearly $p \in M_{t}$ for some $t \in \mathbf{R}$. Choose some open subset $\mathcal{C}_{*}$ of $M_{t}$ with $p \in \mathcal{C}_{*}$ such that $\mathcal{C}_{*}$ has compact closure and $\partial \mathcal{C}_{*}$ is a smooth, two-dimensional 
submanifold of $M_{t}$. By the last Theorem, $\mathcal{R}_{\omega}\left(\diamond\left(\mathcal{C}_{*}\right)\right)$ is a factor. Let $(\mathbf{k}, \mathcal{H})$ be the one-particle Hilbert-space structure of $\omega$ and set $\mathcal{L}:=\mathbf{k}\left(K_{\mathcal{C}_{*}}\right), \mathcal{L}_{p}:=\left(K_{\left(\mathcal{C}_{*} \backslash\{p\}\right)}\right)$. Then we have

$$
W^{F}\left(\mathcal{L}_{p}\right)^{\prime \prime} \subset \mathcal{R}_{\omega}(p)^{\prime}
$$

To see this, let $\mathcal{C}_{0}$ be any open subset of $\mathcal{C}_{*}$ containing $p$ and such that $\overline{\mathcal{C}_{0}} \subset \mathcal{C}_{*}$, and set $\mathcal{L}_{0}:=\mathbf{k}\left(K_{\mathcal{C}_{*}}\right)$. Then obviously

$$
\mathcal{R}_{\omega}(p) \subset \mathcal{R}_{\omega}\left(\diamond\left(\mathcal{C}_{0}\right)\right)=W^{F}\left(\mathcal{L}_{0}\right)^{\prime \prime}
$$

From the results of [Ara] or [LRT] one obtains

$$
W^{F}\left(\mathbf{k}\left(K_{\left(\mathcal{C}_{*} \backslash \overline{\mathcal{C}_{0}}\right)}\right)\right)^{\prime \prime} \subset W^{F}\left(\mathcal{L}_{0}^{\mathbf{v}}\right)^{\prime \prime}=\mathcal{R}_{\omega}\left(\diamond\left(\mathcal{C}_{0}\right)\right)^{\prime} \subset \mathcal{R}_{\omega}(p)^{\prime}
$$

where $\mathbf{k}\left(K_{\left(\mathcal{C}_{*} \backslash \overline{\left.\mathcal{C}_{0}\right)}\right.}\right) \subset \mathcal{L}_{0}^{\mathbf{v}}$ can be shown as in the last theorem. On the other hand one has that $W^{F}\left(\mathcal{L}_{p}\right)^{\prime \prime}$ is the von Neumann algebra generated by the family of sets $W^{F}\left(\mathbf{k}\left(K_{\left(\mathcal{C}_{*} \backslash \overline{\mathcal{C}_{0}}\right.}\right)\right)$, the $\mathcal{C}_{0}$ ranging over the open subsets of $\mathcal{C}_{*}$ with $\overline{\mathcal{C}_{*}} \subset \mathcal{C}_{*}$ and $p \in \mathcal{C}_{0}$. Thus using von Neumann's density theorem, (4.2) implies (4.1). As $p \in S$ for some smooth, compact, two-dimensional submanifold $S$ of $\mathcal{C}_{*}$ and $K_{\left(\mathcal{C}_{*} \backslash S\right)}$ is dense in $K_{\mathcal{C}_{*}}$ with respect to the topology given by $\mu_{\omega}$ (see below where this is proved for the canonical vacuum state and use Proposition 3.8(c)), one obtains that $\mathcal{L}_{p}$ is dense in $\mathcal{L}$ in the topology of $\mathcal{H}$. Then $W^{F}(\mathcal{L})^{\prime \prime}=W^{F}\left(\mathcal{L}_{p}\right)^{\prime \prime} \subset \mathcal{R}_{\omega}(p)^{\prime}$, implying $\mathcal{R}_{\pi}(p) \subset W^{F}(\mathcal{L})^{\prime}$. On the other hand, $\mathcal{R}_{\omega}(p) \subset W^{F}(\mathcal{L})^{\prime \prime}=\mathcal{R}_{\omega}\left(\diamond\left(\mathcal{C}_{*}\right)\right)$, and so we have by the last theorem,

$$
\mathcal{R}_{\omega}(p) \subset \mathcal{R}_{\omega}\left(\diamond\left(\mathcal{C}_{*}\right)\right) \cap \mathcal{R}_{\omega}\left(\diamond\left(\mathcal{C}_{*}\right)\right)^{\prime}=\mathbf{C 1},
$$

as desired.

Remark. From the proof it also follows that

$$
\bigcap_{\mathcal{O} \supset S} \mathcal{R}_{\omega}(\mathcal{O})=\mathbf{C 1}
$$

if $S$ is compact and contained in the union of finitely many smooth, closed, twodimensional submanifolds of any of the $M_{t}, t \in \mathbf{R}$.

Now we can make use of a result of Wollenberg (see [Wol], or Sect. 16.2.4 and Theorem 16.2.18 in [BW]) which implies that if $\omega$ is a state possessing scaling limits and satisfying local definiteness at all spacetime points, then $\mathcal{R}_{\omega}(\mathcal{O})$ is, provided it is a factor, of type $I I I_{1}$ for sufficiently small, specifically shaped spacetime regions $\mathcal{O}$. Quasifree Hadamard states on $\mathcal{A}[K, \kappa]$ possess scaling limits at all points in spacetime and so we arrive at the following

Proposition 4.3. Let $\omega$ be a quasifree, Hadamard state on the Weyl-algebra of the KG-field in the ultrastatic, globally hyperbolic spacetime $(\mathcal{M}, g)$. Given any point $p$ in $\mathcal{M}$, every neighbourhood $\tilde{\mathcal{O}}$ of p possesses an open sub-neighbourhood $\mathcal{O}$ of $p$ such that $\mathcal{R}_{\omega}(\mathcal{O})$ is a factor of type $I I I_{1}$.

Remark. In general, the regions $\mathcal{O}$ for which the assertion of the last proposition has been made may be very small. In any case, the open interior of their causal complement must be non-void.

We still have to show that there is a pure, quasifree Hadamard state with the denseness-property as required in the proofs of the last two theorems. This will 
be the "canonical vacuum state" $\hat{\omega}$ on the Weyl-algebra of the KG-field in the ultrastatic spacetime $(\mathcal{M}, g)$ foliated by the complete, three-dimensional Riemannian manifold $(M, \gamma)$. We shall exhibit a one-parameter family of one-particle Hilbert-space structures for $\hat{\omega}$. To do this, we need the following

Theorem 4.4 Let $\nu$ be the measure induced by $\gamma$ on $M$ and $\Delta_{\gamma}$ the Laplace-Beltrami operator for $(M, \gamma)$. Then the operator

$$
-\Delta_{\gamma}+m^{2}: \mathcal{D}(M) \rightarrow L^{2}(M, \nu)
$$

is essentially selfadjoint in $L^{2}(M, \nu)$; its closure, denoted by $A$, is strictly positive and hence invertible.

Proof. This is proved in [Che]. See also [Kay1].

Now let us define the following family $\left(\mathbf{k}^{t}, \mathcal{H}^{t}\right), t \in \mathbf{R}$, of one-particle Hilbertspace structures over the symplectic space $(K, \kappa)$ :

$$
\begin{gathered}
\mathcal{H}^{t}:=L_{\mathbf{C}}^{2}(M, \nu), \\
\mathbf{k}^{t}[f]:=\frac{1}{\sqrt{2}}\left(A^{1 / 4}\left(\varrho_{0}^{t} E f\right)+i A^{-1 / 4}\left(\varrho_{1}^{t} E f\right)\right) \quad \forall[f] \in K,
\end{gathered}
$$

where $E$ is the propagator of the KG-Eq. and we define for $t \in \mathbf{R}$ and $\psi \in \mathcal{E}(\mathcal{M})$ :

$$
\begin{aligned}
& \varrho_{0}^{t} \psi:=\psi \mid M_{t} \in \mathcal{D}(M), \\
& \varrho_{1}^{t} \psi:=\left(\partial_{t} \psi\right) \mid M_{t} \in \mathcal{D}(M) .
\end{aligned}
$$

Here $M_{t}, t \in \mathbf{R}$, is the natural foliation of $\mathcal{M}$, and $M$ and $M_{t}$ are canonically identified. Then one arrives at the following

Theorem 4.5. For each $t \in \mathbf{R},\left(\mathbf{k}^{t}, \mathcal{H}^{t}\right)$ is a one-particle structure for the unique quasifree state $\hat{\omega}$ on the Weyl-algebra $\mathcal{A}[K, \kappa]$ of the $\mathrm{KG}$-field in $(\mathcal{M}, g)$ which is a ground state for the time-translations. $\hat{\omega}$ is called the canonical vacuum. It is a pure state, and its spatio-temporal two-point function is of Hadamard form.

Proof. See [Kay1] for the proof that the family of one-particle structures belongs to a single pure ground state. The uniqueness of quasifree ground states in the case that the spectrum of the generator of the time-translations is strictly positive (this is the case here, cf. [Kay1]) is proved in [Kay4]. See [FNW; KW] for the proof that the canonical vacuum is an Hadamard state.

Now we state the promised denseness-property.

Proposition 4.6. Let $t \in \mathbf{R}$, and $\mathcal{C}_{*}$ an open subset of $M_{t}$ with compact closure such that $\partial \mathcal{C}_{*}$ is contained in the union of finitely many smooth, closed, two-dimensional submanifolds of $M_{t}$. Then $K_{\mathcal{C}_{*}}+K_{M_{t} \backslash \overline{\mathcal{C}_{*}}}=K_{\left(M_{t} \backslash \partial \mathcal{C}_{*}\right)}$ is dense in $K$ in the norm given by $\mu_{\hat{\omega}}$ on $K$.

Proof. We have

$$
\mu_{\hat{\omega}}([f],[f])=\left\langle\mathbf{k}^{t}[f], \mathbf{k}^{t}[f]\right\rangle_{\mathcal{H}^{t}} \quad \forall[f] \in K .
$$

On the other hand, if $T_{M_{t}}$ is the symplectomorphism of Proposition 2.2, we have

$$
2\left\|\mathbf{k}^{t} \circ T_{M_{t}}^{-1}\left(u_{0}, u_{1}\right)\right\|_{\mathcal{H}^{t}}^{2}=\left\|A^{1 / 4} u_{0}\right\|_{L^{2}}^{2}+\left\|A^{-1 / 4} u_{1}\right\|_{L^{2}}^{2}
$$


for all $\left(u_{0}, u_{1}\right) \in \mathbf{D}_{M_{t}}$, where the norms on the right-hand side are given by the scalar product on $L_{2}(M, \nu)$. Since

$$
K_{\left(M_{t} \backslash \partial \mathcal{C}_{*}\right)}=T_{M_{t}}^{-1}\left(\mathbf{D}_{\left(M_{t} \backslash \partial \mathcal{C}_{*}\right)}\right),
$$

the proposition is proved if $\mathbf{D}_{\left(M_{t} \backslash \partial \mathcal{C}_{*}\right)}$ is dense in $\mathbf{D}_{M_{t}}$ with respect to the topology given by the right-hand side of (4.3). $A^{-1 / 4}$ is a bounded operator on $L^{2}(M, \nu)$ and so $C_{0}^{\infty}\left(M_{t} \backslash \partial \mathcal{C}_{*}\right)$ is dense in $C_{0}^{\infty}\left(M_{t}\right)$ with respect to the topology given by $\left\|A^{-1 / 4} \cdot\right\|_{L^{2}}$. It is far less obvious but also true that $C_{0}^{\infty}\left(M_{t} \backslash \partial \mathcal{C}_{*}\right)$ is dense in $C_{0}^{\infty}\left(M_{t}\right)$ with respect to the topology given by $\left\|A^{1 / 4} \cdot\right\|_{L^{2}}$. (A proof of this will be given in Appendix $\mathrm{D}$.) This shows that $\mathbf{D}_{\left(M_{t} \backslash \partial \mathcal{C}_{*}\right)}$ is dense in $\mathbf{D}_{M_{t}}$ with respect to the topology given by the right-hand side of (4.3), and so the proposition is proved.

Acknowledgements. Major parts of the results of this work are taken from the author's Diploma Thesis written at the Institute for Theoretical Physics, Technische Universität Berlin. The author wishes to express his thanks to K.-E. Hellwig for supervision and advice, and also to R. Seiler. Thanks are also extended to K. Fredenhagen and B. S. Kay for helpful conversations and comments. The author is particularly indebted to M. Keyl, M. Köhler and M. Wollenberg for many useful hints and discussions. Financial support by the Siegfried Roggenbuck-Stiftung through a study grant is also gratefully acknowledged.

\section{Appendix A}

Proof of Lemma 3.7. Since $\mathcal{C}$ is a Cauchy-surface for $\mathcal{N}, \mathcal{N}$ is a globally hyperbolic sub-spacetime of $(\mathcal{M}, g)$ and thus, since the closure of $G$ is a compact subset of $\mathcal{N}$, there are two Cauchy-surfaces $\mathcal{C}^{+}, \mathcal{C}^{-}$in $\mathcal{N}$ such that $\mathcal{C}$ and $G$ are contained in $\mathcal{L}:=I^{+}\left(\mathcal{C}^{-}\right) \cap I^{-}\left(\mathcal{C}^{+}\right)^{2}$. We set $\tilde{G}:=J(\bar{G}) \cap \mathcal{C}$ and note that $D(\tilde{G}) \cap \overline{\mathcal{L}}$ is a compact subset of $\mathcal{N}$ containing $G$, since $\tilde{G}$ is a compact subset of $\mathcal{C}$. Let $\hat{G}$ be compact subset of $\mathcal{C}$ such that $\tilde{G}$ is fully contained in the open interior of $\hat{G}$. Then $I(\hat{G}) \cap \mathcal{N}$ is a neighbourhood of $J(\tilde{G}) \cap \mathcal{N}$. We introduce the following open covering of $\mathcal{N}$ : $\mathcal{N}^{0}:=\mathcal{N} \backslash J(\tilde{G}), \mathcal{N}^{+}:=I(\hat{G}) \cap I^{+}\left(\mathcal{C}^{-}\right) \cap \mathcal{N}, \mathcal{N}^{-}:=I(\hat{G}) \cap I^{-}\left(\mathcal{C}^{+}\right) \cap \mathcal{N}$. By $\psi^{0}$, $\psi^{+}, \psi^{-}$we denote a partition of unity (in $\mathcal{N}$ ) subordinate to that covering, and define

$$
P^{M N}(p, q):=\psi^{M}(p) \psi^{N}(q) B(p, q)
$$

for all $p, q \in \mathcal{N}$ and $M, N \in\{+,-\}$. We also use the notation

$$
\bar{M}:= \begin{cases}- & \text { if } M=+ \\ + & \text { if } M=-\end{cases}
$$

for $M \in\{+,-\}, \bar{N}$ is defined accordingly. Then one checks, using the support properties of $\psi^{+}, \psi^{-}$, that the following holds:

$$
\begin{aligned}
& \left(\nabla^{a} \nabla_{a}+m^{2}\right)_{p}\left(\nabla^{a} \nabla_{a}+m^{2}\right)_{q} P^{++}(p, q) \\
& \quad=-\left(\nabla^{a} \nabla_{a}+m^{2}\right)_{p}\left(\nabla^{a} \nabla_{a}+m^{2}\right)_{q} P^{-+}(p, q) \\
& \quad=-\left(\nabla^{a} \nabla_{a}+m^{2}\right)_{p}\left(\nabla^{a} \nabla_{a}+m^{2}\right)_{q} P^{+-}(p, q) \\
& \quad=\left(\nabla^{a} \nabla_{a}+m^{2}\right)_{p}\left(\nabla^{a} \nabla_{a}+m^{2}\right)_{q} P^{--}(p, q)
\end{aligned}
$$

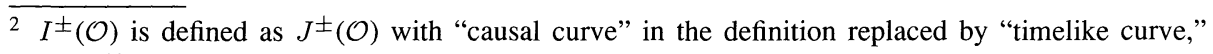
see [Wal3] 
for all $p, q \in J(\tilde{G}) \cap \mathcal{N}$; and if $f, h \in \mathcal{D}(G)$, then one has with $M, N \in\{+,-\}$,

$$
E^{M} f(p) E^{N} h(q) P^{\bar{M} \bar{N}}(p, q)=0
$$

for $p$ or $q$ in $\mathcal{N}^{0} \cup J^{+}\left(\mathcal{C}^{+}\right) \cup J^{-}\left(\mathcal{C}^{-}\right)$, where $E^{+}$and $E^{-}$are the advanced and retarded fundamental solutions of the KG-Eq. Also, we have that $E^{M} f$ and its normal derivatives vanish on $\mathcal{C}^{\bar{M}}$ for $M \in\{+,-\}$ and if $f \in \mathcal{D}(G)$; further, $P^{\bar{M} \bar{N}}(p, q)$ and its normal derivatives with respect to $p(q)$ on $\mathcal{C}^{M}\left(\mathcal{C}^{N}\right)$ vanish for $p \in \mathcal{C}^{M}$ or $q \in \mathcal{C}^{N}$, $M, N=+,-$. We make use of these properties applying Green's identity

$$
\begin{gathered}
\int_{\mathcal{L}}\left(u\left(\nabla^{a} \nabla_{a}+m^{2}\right) v-v\left(\nabla^{a} \nabla_{a}+m^{2}\right) u\right) d \eta \\
=\int_{\partial \mathcal{L}}\left(u n^{a} \nabla_{a} v-v n^{a} \nabla_{a} u\right) d \eta_{(\partial \mathcal{L})}
\end{gathered}
$$

with respect to the variables $p$ and $q$, yielding

$$
\begin{aligned}
& \int_{\mathcal{L} \times \mathcal{L}} E^{M} f(p) E^{N} h(q)\left(\nabla^{a} \nabla_{a}+m^{2}\right)_{p}\left(\nabla^{a} \nabla_{a}+m^{2}\right)_{q} P^{\bar{M} \bar{N}}(p, q) d \eta(p) d \eta(q) \\
& \quad=\int_{\mathcal{L} \times \mathcal{L}} f(p) h(q) P^{\bar{M} \bar{N}}(p, q) d \eta(p) d \eta(q)
\end{aligned}
$$

for all $f, h \in \mathcal{D}(G)$ and $M, N=+,-$, since the boundary terms vanish by what we just remarked. Since the integrands in (A.3) are compactly supported in $\mathcal{L} \times \mathcal{L}$, we may replace the integration region $\mathcal{L} \times \mathcal{L}$ by $\mathcal{N} \times \mathcal{N}$. Now observe that by (A.1) and the support properties of $E^{ \pm}$one obtains

$$
\begin{aligned}
& E f(p) E h(q)\left(\nabla^{a} \nabla_{a}+m^{2}\right)_{p}\left(\nabla^{a} \nabla_{a}+m^{2}\right)_{p} P^{--}(p, q) \\
& =\sum_{M, N=+,-} E^{M} f(p) E^{N} h(q) P^{\bar{M}} \bar{N}(p, q)
\end{aligned}
$$

for all $f, h \in \mathcal{D}(G)$ and $p, q \in \mathcal{N}$, implying with (A.1-3)

$$
\begin{aligned}
& \int_{\mathcal{N} \times \mathcal{N}} \operatorname{Ef}(p) E h(q)\left(\nabla^{a} \nabla_{a}+m^{2}\right)_{p}\left(\nabla^{a} \nabla_{a}+m^{2}\right)_{q} P^{--}(p, q) d \eta(p) d \eta(q) \\
& \quad=\int_{\mathcal{N} \times \mathcal{N}} f(p) h(q) B(p, q) d \eta(p) d \eta(q)
\end{aligned}
$$

for $f, h \in \mathcal{D}(G)$. The integrand of the first integral of (A.5) vanishes for all $f, h \in \mathcal{D}(G)$ outside of the Cartesian product of the set $J(\tilde{G}) \cap \overline{\mathcal{L}}$ which is compact in $\mathcal{N}$, so if we choose a smooth function $\xi$ compactly supported on $\mathcal{N} \times \mathcal{N}$ such that $\xi \equiv 1$ on the Cartesian product of $J(\tilde{G}) \cap \mathcal{L}$, then the function

$$
Q(p, q):=\xi(p, q)\left(\nabla^{a} \nabla_{a}+m^{2}\right)_{p}\left(\nabla^{a} \nabla_{a}+m^{2}\right)_{q} P^{--}(p, q)
$$

is a function in $C_{0}^{\infty}(\mathcal{N} \times \mathcal{N})$ having the property as claimed in the statement of the lemma. 


\section{Appendix B}

Representation of Elements in $\mathcal{D}\left(\mathcal{O}_{1} \times \mathcal{O}_{2}\right)$ for Open Sets $\mathcal{O}_{1} \subset \mathbf{R}^{k}, \mathcal{O}_{2} \subset \mathbf{R}^{l}$

In this section we give a proof of the denseness of the algebraic tensorproduct $\mathcal{D}\left(\mathcal{O}_{1}\right) \otimes \mathcal{D}\left(\mathcal{O}_{2}\right)$ in $\mathcal{D}\left(\mathcal{O}_{1} \times \mathcal{O}_{2}\right)$ which differs from the original proof in [S] in giving a more detailed description of sequences in $\mathcal{D}\left(\mathcal{O}_{1}\right) \otimes \mathcal{D}\left(\mathcal{O}_{2}\right)$ approaching given elements in $\mathcal{D}\left(\mathcal{O}_{1} \times \mathcal{O}_{2}\right)$. Namely, let $f \in \mathcal{D}\left(\mathcal{O}_{1} \times \mathcal{O}_{2}\right)$. Then there are sequences $\phi_{j} \in \mathcal{D}\left(\mathcal{O}_{1}\right)$, $\psi_{\jmath} \in \mathcal{D}\left(\mathcal{O}_{2}\right), j \in \mathbf{N}$, with $\sum_{j=1}^{n} \phi_{\jmath} \otimes \psi_{j} \rightarrow f$ for $n \rightarrow \infty$ in the topology of $\mathcal{D}\left(\mathcal{O}_{1} \times \mathcal{O}_{2}\right)$ such that

$$
\begin{gathered}
\sum_{j \in \mathbf{N}} p\left(\phi_{\jmath} \otimes \phi_{j}\right)^{\lambda} p\left(\psi_{j} \otimes \psi_{j}\right)^{\lambda}<\infty \\
\sum_{j \in \mathbf{N}} p\left(\phi_{\jmath} \otimes \psi_{\jmath}\right)^{\lambda}<\infty
\end{gathered}
$$

for all $\lambda>0$ and every seminorm $p$ in the family of seminorms defining the topology of $\mathcal{D}\left(\mathcal{O}_{1} \times \mathcal{O}_{2}\right)$. To see this, let us define $I:=(-\pi, \pi)$ and assume that $f$ has compact support in $I^{k} \times I^{l}$. The general case then follows by using a scaling argument. We set, for $\nu \in \mathbf{N}$ and $\tau \in I$,

$$
\begin{aligned}
e_{2 \nu-1}(\tau) & :=\frac{1}{\sqrt{\pi}} \cos (\nu \tau), \\
e_{2 \nu}(\tau) & :=\frac{1}{\sqrt{\pi}} \sin (\nu \tau), \\
e_{0}(\tau) & :=\frac{1}{\sqrt{\pi}} .
\end{aligned}
$$

Now choose some smooth function $\chi$ compactly supported on $I$ and equal to unity on a large enough sub-interval of $I$ so that the $(k+l)$-fold tensorproduct of $\chi$ is equal to unity on $\operatorname{supp}(f)$, and define for multiindices $m \in \mathbf{N}_{0}^{k}$ and $n \in \mathbf{N}_{0}^{l}$;

$$
\begin{aligned}
\xi_{m} & :=\xi e_{m_{1}} \otimes \ldots \otimes \chi e_{m_{k}}, \\
\theta_{m} & :=\xi e_{n_{1}} \otimes \ldots \otimes \chi e_{n_{l}} .
\end{aligned}
$$

Then the Fourier-coefficients of $f$,

$$
\alpha_{m n}:=\int f(x, y) \xi_{m}(x) \theta_{n}(y) d^{k} x d^{l} y
$$

have the property to fall off more rapidly than the inverse of any polynomial in the $m, n$ for large $|m|,|n|$. More precisely, using the usual multiindex notation, it holds that

$$
\left|\alpha_{m n}\right| m^{\beta} n^{\varrho} \rightarrow 0 \text { for }|m|+|n| \rightarrow \infty
$$

for every pair of multiindex $\beta \in \mathbf{N}^{k}, \varrho \in \mathbf{N}^{l}$. This can be seen from the fact that $f \in \mathcal{D}\left(I^{k} \times I^{l}\right)$ by using integration by parts inductively. On the other hand, if we set for $\beta \in \mathbf{N}_{0}^{k}, \varrho \in \mathbf{N}_{0}^{l}$

$$
p_{(\beta, \varrho)}(F):=\sup _{(x, y) \in I^{k} \times I^{l}}\left|\partial_{x}^{\beta} \partial_{y}^{\varrho} F(x, y)\right|
$$


for all $F \in \mathcal{D}\left(I^{k} \times I^{l}\right)$, then it is easily seen that

$$
\begin{aligned}
& p_{(\beta, \varrho)}\left(\xi_{m} \otimes \xi_{n}\right) \leq C_{1}|m|^{k|\beta|}|m|^{k|\varrho|}, \\
& p_{(\beta, \varrho)}\left(\theta_{n} \otimes \theta_{n}\right) \leq C_{2}|n|^{l|\beta|}|n|^{l|\varrho|}, \\
& p_{(\beta, \varrho)}\left(\xi_{m} \otimes \theta_{n}\right) \leq C_{3}|m|^{k|\beta|}|n|^{l|\varrho|}
\end{aligned}
$$

for some positive constants $C_{i}, i=1,2,3$, depending on $\beta$ and $\varrho$. Therefore, choosing a denumeration $j \mapsto\left(m_{\jmath}, n_{\jmath}\right), j \in \mathbf{N}$ of the set of multiindex-pairs $m \in \mathbf{N}_{0}^{k}, n \in \mathbf{N}_{0}^{l}$, and defining $\phi_{j}:=\alpha_{m_{\jmath} n_{\jmath}} \xi_{m_{\jmath}}, \psi_{\jmath}:=\theta_{n_{\jmath}}, j \in \mathbf{N}$, one obtains that

$$
\sum_{j=1}^{N} \phi_{\jmath} \otimes \psi_{\jmath} \rightarrow f(N \rightarrow \infty) \quad \text { in } \mathcal{D}\left(I^{k} \times I^{l}\right)
$$

and (B.1) and (B.2) follow from the rapid decrease property of the $\alpha_{m n}$.

\section{Appendix C}

\section{Hadamard States}

In this Appendix we introduce the notion of Hadamard states on the Weyl-algebra $\mathcal{A}[K, \kappa]$ of the KG-Field in a globally hyperbolic spacetime. We present parts of the discussion by Kay and Wald [KW] and hence our Hadamard states will be globally Hadamard states. (There is a related, weaker, local Hadamard condition which one could impose. This is discussed in [GK; Kay5], where it is conjectured that the weaker, local Hadamard condition for states of the KG-field already implies the global Hadamard condition as we quote it here from [KW]. A resolution of this conjecture, showing it to be true, has recently been presented [Rad]. We refer to the cited literature for further details.)

First we define what it means for a bilinear functional on $\mathcal{D}(\mathcal{M}) \times \mathcal{D}(\mathcal{M})$ with values in $\mathbf{C}$ to be of Hadamard form. To start with this task, let $\mathcal{X}$ be the subset of those $(p, q) \in \mathcal{M} \times \mathcal{M}$ which are causally related and are such that $J^{+}(p) \cap J^{-}(q)$ and $J^{+}(q) \cap J^{-}(p)$ are contained within a causal normal neighbourhood. Then $s(p, q)$, the squared geodesic distance from $p$ to $q$, is well-defined and smooth in an open neighbourhood $\Omega$, in $\mathcal{M} \times \mathcal{M}$, of $\mathcal{X}$. Let the functions $U$ and $V_{m}, m \in \mathbf{N}_{0}$, defined on $\Omega$, denote the square root of the VanVleck-Morette determinant, and the sequence determined by the Hadamard recursion relations, respectively ${ }^{3}$. Set, for $n \in \mathbf{N}$ and $(p, q) \in \Omega$,

$$
V^{(n)}(p, q):=\sum_{m=0}^{n} V_{m}(p, q)(s(p, q))^{m} .
$$

Let $T: \mathcal{M} \rightarrow \mathbf{R}$ be a smooth time-function increasing towards the future and define for all $\varepsilon>0$ and $(p, q) \in \Omega$,

$$
Q_{T}(p, q ; \varepsilon):=s(p, q)-2 i \varepsilon(T(p)-T(q))-\varepsilon^{2}
$$

\footnotetext{
3 These objects are e.g. defined in [Gar], see also [Fri]. It is important to note that for fixed $m$, the mass term appearing in the KG-Eq., they are determined solely by the spacetime metric $g$
} 
and

$$
G_{\varepsilon}^{T, n}(p, q):=\frac{1}{4 \pi^{2}}\left(\frac{U(p, q)}{Q_{T}(p, q ; \varepsilon)}+V^{(n)}(p, q) \ln \left(Q_{T}(p, q ; \varepsilon)\right)\right)
$$

(ln is the principal branch of the logarithm). Next, let $\mathcal{N}$ be a causal neighbourhood of some Cauchy-surface $\mathcal{C}$. An $\mathcal{N}$-regularizing function $\chi$ is a smooth function on $\mathcal{N} \times \mathcal{N}$ having the following property: There is an open neighbourhood, $\Omega_{*}$, in $\mathcal{N} \times \mathcal{N}$ of the set of pairs of causally related points in $\mathcal{N}$ such that $\overline{\Omega_{*}} \subset \Omega$, and $\chi \equiv 1$ on $\Omega_{*}$ and $\chi \equiv 0$ outside of $\Omega$. It is not difficult to see that $\mathcal{N}$-regularizing functions always exist.

Definition C.1. Let $\Lambda$ be a linear form on $\mathcal{D}(\mathcal{M}) \times \mathcal{D}(\mathcal{M})$ with values in $\mathbf{C}$. We say that $\Lambda$ is of Hadamard form if there exist

(i) a causal normal neighbourhood $\mathcal{N}$ of some Cauchy-surface $\mathcal{C}$,

(ii) an $\mathcal{N}$-regularizing function $\chi$,

(iii) a smooth time-function $T$ on $\mathcal{M}$ increasing towards the future,

(iv) a sequence $H^{n} \in C^{n}(\mathcal{N} \times \mathcal{N})$

such that

$$
\Lambda(f, h)=\lim _{e \rightarrow 0+} \int \Lambda_{\varepsilon}^{T, n}(p, q) f(p) H(q) d \eta(p) d \eta(q)
$$

for all $f, h \in \mathcal{D}(\mathcal{N})$ and all $n \in \mathbf{N}$, where

$$
\Lambda_{\varepsilon}^{T, n}(p, q):=\chi(p, q) G_{\varepsilon}^{T, n}(p, q)+H^{n}(p, q) .
$$

The following results show that this is in fact a reasonable definition.

Proposition C.2. (a) The definition of the Hadamard form is independent of the choice of $\mathcal{C}, \mathcal{N}, T$ and $\chi$. This implies in particular that, if $\Lambda$ is of Hadamard form in a causal normal neighbourhood $\mathcal{N}$ of some Cauchy-surface $\mathcal{C}$ (in the sense that (C.1) holds for $f, h \in \mathcal{D}(\mathcal{N})$ ), then it is of Hadamard form in some causal normal neighbourhood $\mathcal{N}^{\prime}$ of any other Cauchy-surface $\mathcal{C}^{\prime}$. This behaviour is called the "causal propagation property" of the Hadamard form.

(b) If $\Lambda$ is of Hadamard form, then it is locally a distribution. This means that for every $p \in \mathcal{M}$ there is a neighbourhood $\mathcal{O}$ of $p$ such that the real and imaginary parts of $\Lambda$ can be identified with elements of $\mathcal{D}^{\prime}(\mathcal{O} \times \mathcal{O})$.

Proof. See [FSW; KW] for (a). Part (b) of the Proposition follows from Appendix B in [KW]. (To see this, replace on the right-hand side of Eq. (B.8) in [KW] the function $F_{2}\left(x_{2}\right)$ by a smooth function $F\left(x_{1}, x_{2}\right)$ and deduce - using the same arguments as given for $F_{2}\left(x_{2}\right)$ - that the resulting family of integrals converges for $\varepsilon \rightarrow 0$ to a smooth function in $x_{1}$ if $F$ is supported in a small enough region $\mathcal{O} \times \mathcal{O}$. So the limit (C.1) exists if $f \otimes h$ is replaced by $F \in \mathcal{D}(\mathcal{O} \times \mathcal{O})$. Then use that weak limits of sequences of distributions (which are here given through the families of class $C^{n}$ integral kernels $\Lambda_{\varepsilon}^{T, n}, \varepsilon>0$ for arbitrary, but fixed $n$ ) are again distributions.)

Definition C.3. Let $\omega$ be a state on the Weyl-algebra $\mathcal{A}[K, \kappa]$ of the KG-field in the globally hyperbolic spacetime $(\mathcal{M}, g)$. Then $\omega$ is called an Hadamard state if its twopoint function exists and if its spatio-temporal two-point distribution is of Hadamard form.

The next result shows that the set of Hadamard states which are also quasifree is large. 
Theorem C.4. The set of quasifree Hadamard states on $\mathcal{A}[K, \kappa]$ spans an infinitedimensional sub-vectorspace of the topological dual space of $\mathcal{A}[K, \kappa]$.

Proof. Adopting the methods of [FNW] (cf. [KW]) one obtains the existence of quasifree Hadamard states. Now let $\omega$ be a quasifree Hadamard state and $\mu_{\omega}$ the associated real scalar product on $K$. It is easily seen that we can find a sequence $\phi_{j} \in \mathcal{D}(\mathcal{M}), j \in \mathbf{N}$, such that the $\left[\phi_{j}\right]$ form an orthonormal system in $K$ with respect to $\mu_{\omega}$. We define a sequence of real scalar products

$$
\mu_{j}(x, y):=\mu_{\omega}(x, y)+\mu_{\omega}\left(x,\left[\phi_{j}\right]\right) \mu_{\omega}\left(\left[\phi_{j}\right], y\right) \quad \forall x, y \in K
$$

on $K$. Notice that $\mu_{j}(x, x) \geq \mu_{\omega}(x, x)$ so that the $\mu_{j}$ give rise to quasifree states $\omega_{j}$ on $\mathcal{A}[K, \kappa]$. It follows from the results of [KW] that near Cauchy-surfaces, the spatiotemporal two-point functions $\Lambda_{j}$ of the $\omega_{j}$ differ from $\Lambda_{\omega}$ by a smooth bi-solution, hence the $\omega_{j}$ are quasifree Hadamard states. The linear independence is obtained by inspection.

Let $\omega_{j}, j=1,2$, be two quasifree, Hadamard states on $\mathcal{A}[K, \kappa]$ with associated real scalar products $\mu_{j}$ on $K$, and spatio-temporal two-point distributions $\Lambda_{j}$. Then the difference of the $\Lambda_{j}$ is, near Cauchy-surfaces, a smooth, real-valued bi-solution of the KG-Eq., i.e. if $\mathcal{N}$ is a causal normal neighbourhood of some Cauchy-surface $\mathcal{C}$ in $(\mathcal{M}, g)$, then

$$
\Lambda_{1}(f, h)-\Lambda_{2}(f, h)=\int f(p) B(p, q) h(q) d \eta(p) d \eta(q)
$$

for all $f, h \in \mathcal{D}(\mathcal{N})$, where $B \in C^{\infty}(\mathcal{N} \times \mathcal{N})$ is a bi-solution,

$$
\left(\nabla^{a} \nabla_{a}+m^{2}\right)_{p} B(p, q)=0=\left(\nabla^{a} \nabla_{a}+m^{2}\right)_{q} B(p, q)
$$

for all $p, q \in \mathcal{N}$. This is easily seen from the definition of the Hadamard form and the fact that $\Lambda$, are weak bi-solutions of the $\mathrm{KG}$-Eq. as remarked at the beginning of Sect. 3, (C.3) then results from applying Green's identity. That $B$ is real-valued stems from the fact that

$$
\Lambda_{1}(f, h)-\Lambda_{2}(f, h)=\mu_{1}([f],[h])-\mu_{2}([f],[h])
$$

for all $f, h \in \mathcal{D}(\mathcal{M})$ : The right-hand side is always real-valued.

\section{Appendix D}

Proposition D.1. With the assumptions and notation as in Sect. 4 , let the compact set $S$ be contained in the union $\tilde{S}=\bigcup_{i=1}^{l} S_{i}$ of finitely many smooth, closed, two-dimensional submanifolds of $M$, and let

$$
\|f\|_{A}^{2}:=\left\langle A^{1 / 4} f, A^{1 / 4} f\right\rangle_{L^{2}} \quad \forall f \in \mathcal{D}(M) .
$$

Then $\mathcal{D}(M \backslash S)$ is dense in $\mathcal{D}(M)$ with respect to $\|\cdot\|_{A}$.

Proof. Around every point $p \in M$ there is a chart $\Psi=\left(x^{i}\right)$ with the property

$$
\operatorname{det}\left(\gamma_{i j}\right) \equiv 1 \text { on } \operatorname{dom}(\Psi)
$$

(see Theorem 12.5 in [CFKS]). So choose for every point $p \in S$ a chart $\Psi_{p}$ with the property (D.1), and an open neighbourhood $U_{p}$ of $p$ whose closure we require to be 
compact and contained in $\operatorname{dom}\left(\Psi_{p}\right)$. Now we can cover $S$ by finitely many of the $U_{p}$, which will be denoted by $U_{1}, \ldots, U_{n}$. It is not difficult to see that, if we find that for each $j=1, \ldots, n, \mathcal{D}\left(U_{j} \backslash S\right)$ is dense in $\mathcal{D}\left(U_{j}\right)$ with respect to $\|\cdot\|_{A}$, then this implies that $\mathcal{D}(M \backslash S)$ is dense in $\mathcal{D}(M)$ with respect to $\|\cdot\|_{A}$. So we only have to prove that for $U$ open, with $\bar{U} \subset \operatorname{dom}(\Psi)$, where $\Psi$ is a chart with the property (D.1), it holds that $\mathcal{D}(U \backslash S)$ is dense in $\mathcal{D}(U)$ with respect to $\|\cdot\|_{A}$. To show this, we introduce a smooth function $\chi_{\Psi}$ on $M$ such that $0 \leq \chi_{\Psi} \leq 1, \chi_{\Psi} \equiv 1$ on $U$, and $\chi_{\Psi}$ have compact support in $\operatorname{dom}(\Psi)$. We shall use the usual notation $\Psi^{*} f=f \circ \Psi$, $\Psi_{*} \varphi=\varphi \circ \Psi^{-1}$, write $\chi:=\Psi_{*} \chi_{\Psi}$ and define the following linear operator:

$$
\Sigma: \mathcal{D}\left(\mathbf{R}^{3}\right) \rightarrow L^{2}\left(\mathbf{R}^{3}, d^{3} x\right), \quad f \mapsto \Psi_{*}\left(\chi_{\Psi} A^{1 / 2} \Psi^{*}(\chi f)\right) .
$$

Notice how this operator operates: First, $f \in \mathcal{D}\left(\mathbf{R}^{3}\right)$ is multiplied by $\chi$ yielding a function in $\mathcal{D}(\operatorname{Ran}(\Psi))$. This function is by $\Psi^{*}$ mapped to a function in $\mathcal{D}(\operatorname{dom}(\Psi))$, on which $A^{1 / 2}$ is applied, which obviously makes sense since $\mathcal{D}(\operatorname{dom}(\Psi)) \subset \operatorname{dom}\left(A^{1 / 2}\right)$. The result is a function in $L^{2}(M, \nu)$, which after multiplication by $\chi_{\Psi}$ lies in the domain of $\Psi_{*}$ which maps it back into $L^{2}\left(\mathbf{R}^{3}, d^{3} x\right)$. This shows that $\Sigma$ is well-defined. To establish further properties of $\Sigma$ we introduce the following notation:

$$
\begin{aligned}
\langle\varphi, \psi\rangle_{M} & :=\int_{M} \varphi \psi d \nu, \\
\langle f, h\rangle_{\mathbf{R}^{3}} & :=\int_{\mathbf{R}^{3}} f h d^{3} x ;
\end{aligned}
$$

notice that by the special property (D.1) of our chart we have

$$
\left\langle\Psi^{*} f, \Psi^{*} h\right\rangle_{M}=\langle f, h\rangle_{\mathbf{R}^{3}}
$$

and

$$
\left\langle\Psi_{*} \varphi, \Psi_{*} \psi\right\rangle_{\mathbf{R}^{3}}=\langle\varphi, \psi\rangle_{M}
$$

for all $f, h \in L^{2}\left(\operatorname{Ran}(\Psi), d^{3} x\right)$ and all $\varphi, \psi \in L^{2}(\operatorname{dom}(\Psi), \nu)$. We also define

$$
\Theta: \mathcal{D}\left(\mathbf{R}^{3}\right) \rightarrow L^{2}\left(\mathbf{R}^{3}, d^{3} x\right), \quad f \mapsto \chi \Psi_{*} \circ A \circ \Psi^{*}(\chi f)
$$

and denote by $\langle., .\rangle_{H(m)}, m \in \mathbf{R}$, the $m$ th Sobolov scalar product on $\mathcal{D}_{\mathbf{C}}\left(\mathbf{R}^{3}\right)$, defined by

$$
\langle f, h\rangle_{H(m)}:=\int_{\mathbf{R}^{3}} \hat{f}(k) \overline{\hat{h}(k)}\left(|k|^{2}+1\right)^{m} d^{3} k,
$$

where $\hat{f}$ stands for the Fourier-transform of $f$ and the bar denotes complex conjugation. We denote by $\tau$ the selfadjoint closure of the operator

$$
-\Delta+1: \mathcal{D}\left(\mathbf{R}^{3}\right) \rightarrow L^{2}\left(\mathbf{R}^{3}, d^{3} x\right) .
$$

With this notation, it holds that:

1. $\Sigma$ is symmetric.

2. $\langle\Sigma f, \Sigma f\rangle_{\mathbf{R}^{3}} \leq\langle f, \Theta f\rangle_{\mathbf{R}^{3}} \quad \forall f \in \mathcal{D}\left(\mathbf{R}^{3}\right)$.

3. $\langle f, \Theta f\rangle_{\mathbf{R}^{3}} \leq C\langle f, f\rangle_{H(1)}=C\langle f, \tau f\rangle_{\mathbf{R}^{3}} \quad \forall f \in \mathcal{D}\left(\mathbf{R}^{3}\right)$, for some $C>0$.

The properties 1 and 2 are verified by straightforward calculation using (D.4-5). 3 will be proved as follows: Write $\Theta=\alpha^{i j} \partial_{i} \partial_{j}+\beta^{\jmath} \partial_{j}+r$ (summation over the 
indices $i, j=1,2,3$ implied) where $\alpha^{i j}, \beta^{j}$ and $r$ are functions in $\mathcal{D}\left(\mathbf{R}^{3}\right)$. Now, given $\alpha \in \mathcal{D}\left(\mathbf{R}^{3}\right)$ one finds for all $f \in \mathcal{D}\left(\mathbf{R}^{3}\right), i, j=1,2,3$,

$$
\begin{aligned}
\left|\left\langle f, \alpha \partial_{i} \partial_{j} f\right\rangle_{\mathbf{R}^{3}}\right| & =\left|\int f \alpha \partial_{i} \partial_{\jmath} f d^{3} x\right| \\
& =\left|\int \partial_{i}(\alpha f) \partial_{j} f d^{3} x\right| \\
& =\left|\int\left(\left(\partial_{i} \alpha\right) f+\alpha \partial_{j} f\right) \partial_{j} f d^{3} x\right| \\
& \left.=\mid \int\left(\widehat{\left(\partial_{i} \alpha\right.}\right) * \hat{f}+\hat{\alpha} *\left(\widehat{\partial_{j} f}\right)\right) \widehat{\left(\widehat{\partial_{\jmath} f}\right)} d^{3} x \mid \\
& \left.\left.\leq \| \widehat{\left(\partial_{\imath} \alpha\right.}\right) * \hat{f}\left\|_{L^{2}}\right\| \widehat{\partial_{j} f}\left\|_{L^{2}}+\right\| \hat{\alpha} * \widehat{\left(\partial_{\imath} f\right.}\right)\left\|_{L^{2}}\right\| \widehat{\partial_{j} f} \|_{L^{2}} \\
& \leq\left\|\widehat{\partial_{i} \alpha}\right\|_{L^{1}}\|\hat{f}\|_{L^{2}}\left\|\widehat{\partial_{j} f}\right\|_{L^{2}}+\|\hat{\alpha}\|_{L^{1}}\left\|\widehat{\partial_{\imath} f}\right\|_{L^{2}}\left\|\widehat{\partial_{j} f}\right\|_{L^{2}} \\
& \leq\left(\left\|\widehat{\partial_{\imath} \alpha}\right\|_{L^{1}}+\|\hat{\alpha}\|_{L^{1}}\right)\left(\|f\|_{H(1)}\right)^{2} .
\end{aligned}
$$

The second estimate follows from Young's inequality (see [RS]). Similarly, for $\beta$, $r \in \mathcal{D}\left(\mathbf{R}^{3}\right)$ one obtains for all $f \in \mathcal{D}\left(\mathbf{R}^{3}\right), j=1,2,3$ :

$$
\begin{aligned}
\left|\left\langle f, \beta \partial_{j} f\right\rangle_{\mathbf{R}^{3}}\right| & =\left|\int \hat{\beta} * \hat{f\left(\widehat{\partial_{j} f}\right)} d^{3} k\right| \\
& \leq\|\hat{\beta} * \hat{f}\|_{L^{2}}\left\|\widehat{\partial_{j} f}\right\|_{L^{2}} \\
& \leq\|\hat{\beta}\|_{L^{1}}\|\hat{f}\|_{L^{2}}\left\|\widehat{\partial_{j} f}\right\|_{L^{2}} \\
& \leq\|\hat{\beta}\|_{L^{1}}\left(\|f\|_{H(1)}\right)^{2}
\end{aligned}
$$

and

$$
\left|\langle f, r f\rangle_{\mathbf{R}^{3}}\right| \leq\|r\|_{L^{\infty}}\left(\|f\|_{H(1)}\right)^{2} .
$$

With these estimates, 3 is easily deduced. So we obtain by 2 and 3 :

$$
\langle\Sigma f, \Sigma f\rangle_{\mathbf{R}^{3}} \leq C\left\langle\tau^{1 / 2} f, \tau^{1 / 2} f\right\rangle_{\mathbf{R}^{3}} \quad \forall f \in \mathcal{D}\left(\mathbf{R}^{3}\right)
$$

for some $C>0$. Since $\Sigma$ is symmetric, and $\tau$, and thus also $\tau^{1 / 2}$, is positive and essentially selfadjoint on $\mathcal{D}\left(\mathbf{R}^{3}\right)$, a result by Heinz (cf. Appendix E.2) states that (D.6) implies

$$
\left|\langle f, \Sigma f\rangle_{\mathbf{R}^{3}}\right| \leq \tilde{C}\left\langle f, \tau^{1 / 2} f\right\rangle_{\mathbf{R}^{3}}=\tilde{C}\langle f, f\rangle_{H(1 / 2)}
$$

for all $f \in \mathcal{D}\left(\mathbf{R}^{3}\right)$ with some $\tilde{C}>0$. Since $\chi f=f$ for all $f \in \mathcal{D}(\Psi(U))$, we have

$$
\begin{aligned}
\left\langle A^{1 / 4} \Psi^{*} f, A^{1 / 4} \Psi^{*} f\right\rangle_{M} & =\left\langle\Psi^{*} f, \chi_{\Psi} A^{1 / 2} \Psi^{*}(\chi f)\right\rangle_{M} \\
& =\langle f, \Sigma f\rangle_{\mathbf{R}^{3}} \\
& =\tilde{C}\langle f, f\rangle_{H(1 / 2)}
\end{aligned}
$$

for all $f \in \mathcal{D}(\Psi(U))$ and therefore $\mathcal{D}(U \backslash S)$ is dense in $\mathcal{D}(U)$ with respect to $\|\cdot\|_{A}$ if $\mathcal{D}(\Psi(U \backslash S))$ is dense in $\mathcal{D}(\Psi(U))$ with respect to $\|\cdot\|_{H(1 / 2)}$. This is known to be the case under the assumptions made about $S$ :

Lemma D.2. Let $\mathcal{O} \subset \Omega$ be open subsets of $\mathbf{R}^{3}$, and $S$ a smooth, closed, twodimensional submanifold in $\Omega$ intersecting $\mathcal{O}$, and let $f \in \mathcal{D}(\mathcal{O})$. Then there is a sequence $f_{n} \in \mathcal{D}(\mathcal{O} \backslash S), n \in \mathbf{N}$, which approaches $f$ in $\|\cdot\|_{H(1 / 2)}$. 
Notice that if we have proved this lemma, then it holds also true if we only assume that $S$ is the union $\bigcup_{\imath=1}^{k} S_{\imath}$ of finitely many smooth, closed, two-dimensional submanifolds $S_{i}$ of $\Omega$. To see this, use induction over $k$ : Fix $\mathcal{O}$ as above. For $k=1$ the statement is proven by Lemma D.2. Assume that it is true for some finite number $k$. Then the set $\mathcal{O}_{k}:=\mathcal{O} \backslash\left(\bigcup_{i=1}^{k} S_{i}\right)$ is also an open set contained in $\Omega$, and if $S_{k+1}$ is another smooth, closed, two-dimensional submanifold of $\Omega$, then $\mathcal{D}\left(\mathcal{O}_{k} \backslash S_{k+1}\right)$ is dense in $\mathcal{D}\left(\mathcal{O}_{k}\right)$ with respect to $\|\cdot\|_{H(1 / 2)}$ by Proposition E.1. But by assumption, $\mathcal{D}\left(\mathcal{O}_{k}\right)$ is dense in $\mathcal{D}(\mathcal{O})$ with respect to $\|\cdot\|_{H(1 / 2)}$ and hence we can conclude that $\mathcal{D}\left(\mathcal{O}_{k} \backslash S_{k+1}\right)$ is also dense in $\mathcal{D}(\mathcal{O})$ with respect to $\|\cdot\|_{H(1 / 2)}$.

Proof of Lemma D.2. A family $F_{\lambda} \in \mathcal{D}(\mathcal{O}), 1>\lambda>0$, will be constructed such that $0 \leq F_{\lambda} \leq 1, F_{\lambda} \equiv 1$ in a $\lambda$-dependent neighbourhood of $S \cap \operatorname{supp}(f)$, with $\operatorname{supp}\left(F_{\lambda}\right)$ shrinking to $S$ for $\lambda \rightarrow 0$ and with $\left\|F_{\lambda}\right\|_{H(1 / 2)}$ uniformly bounded for $1>\lambda>0$. From these properties it then follows that the functions $\varphi_{\lambda}:=\left(1-F_{\lambda}\right) f$ are in $\mathcal{D}(\mathcal{O} \backslash S)$ for all $1>\lambda>0$, and clearly for all $h \in \mathcal{D}\left(\mathbf{R}^{3}\right)$ one has

$$
\left\langle\varphi_{\lambda}-f, h\right\rangle_{H(1 / 2)}=\left\langle f F_{\lambda}, h\right\rangle_{H(1 / 2)} \rightarrow 0
$$

for $\lambda \rightarrow 0$, and $\left\|\varphi_{\lambda}\right\|_{H(1 / 2)}$ is uniformly bounded for all $1>\lambda>0$ since multiplication by $\mathcal{D}\left(\mathbf{R}^{3}\right)$-functions is a continuous operation in $H(1 / 2)$, the completion of $\mathcal{D}\left(\mathbf{R}^{3}\right)$ with respect to $\|\cdot\|_{H(1 / 2)}$, cf. Appendix E.3. Whence $\left(\varphi_{\lambda}\right)$ converges weakly to $f$ in $H(1 / 2)$. Then there is (cf. Probl. 4.25 in [Wei]) a subsequence $\left(\varphi_{\lambda_{j}}\right)$ of $\left(\varphi_{\lambda}\right)$ such that the sequence

$$
f_{n}:=\frac{1}{n} \sum_{j=1}^{n} \varphi_{\lambda_{\jmath}}, \quad n \in \mathbf{N}
$$

converges for $n \rightarrow \infty$ in $\|\cdot\|_{H(1 / 2)}$ to $f$, and clearly we have $f_{n} \in \mathcal{D}(\mathcal{O} \backslash S)$ for all $n \in \mathbf{N}$. So all that is left to do is to construct a family $F_{\lambda}$ with the above stated properties. For each $s \in S$ there is a neighbourhood $\mathcal{O}_{s}$ of $S$ in $\Omega$ and a chart $\psi_{s}: \mathcal{O}_{s} \rightarrow \mathcal{V}_{s} \subset \mathbf{R}^{3}$ such that $\mathcal{O}_{s} \cap S=\left\{\psi_{s}^{-1}\left(\xi_{1}, \xi_{2}, 0\right)\right.$ $\left.\left(\xi_{1}, \xi_{2}, \xi_{3}\right) \in \mathcal{V}_{s}\right\}$. We may cover the compact set $S \cap \operatorname{supp}(f)$ by finitely many of such $\mathcal{O}_{s}$, say $\mathcal{O}_{1}, \ldots, \mathcal{O}_{k}$, and we can choose functions $\chi_{1}, \ldots, \chi_{k} \in \mathcal{D}(\mathcal{O})$ with the properties: $0 \leq \chi_{i} \leq 1, \operatorname{supp}\left(\chi_{i}\right) \subset \mathcal{O}_{i}$, and $\Sigma_{\imath} \chi_{i} \equiv 1$ in a neighbourhood $U \subset \mathcal{O}$ of $S \cap \operatorname{supp}(f)$. Now choose a smooth function $h: \mathbf{R}^{3} \rightarrow[0,1]$, $\left(\xi_{1}, \xi_{2}, \xi_{3}\right) \mapsto\left(\xi_{3}\right)$ with the properties: $h \equiv 0$ outside of $\xi_{3} \in(-\delta, \delta)$, and $h \equiv 1$ for $\xi_{3} \in(-\delta+\varepsilon, \delta-\varepsilon)$, for some sufficiently small $\delta>\varepsilon>0$. Set $h_{\lambda}\left(\xi_{3}\right):=h\left(\lambda^{-1} \xi_{3}\right)$ for $\lambda \in(0,1)$. Then define $F_{\lambda}:=\Sigma_{i} \xi_{i}\left(h_{\lambda} \circ \psi_{i}\right), \lambda \in(0,1)$. One checks that these functions have the properties: $F_{\lambda} \equiv 1$ in a neighbourhood $U_{\lambda}$ of $S \cap \operatorname{supp}(f), \operatorname{supp}\left(F_{\lambda}\right)$ shrinks to $S$ as $\lambda \rightarrow 0$, and $F_{\lambda} \in \mathcal{D}(\mathcal{O})$. What remains to be shown is that $\left\|F_{\lambda}\right\|_{H(1 / 2)}$ is uniformly bounded for $\lambda \in(0,1)$. We have

$$
\left\|F_{\lambda}\right\|_{H(1 / 2)} \leq \sum_{i=1}^{k}\left\|\chi_{i}\left(h_{\lambda} \circ \psi_{i}\right)\right\|_{H(1 / 2)} .
$$


Since composition by diffeomorphisms is a continuous operation in Sobolev spaces (see Appendix E.4) there is for each $i$ a $C_{i}>0$ such that

$$
\begin{aligned}
\left\|\chi_{i}\left(h_{\lambda} \circ \psi_{i}\right)\right\|_{H(1 / 2)} & =\left\|\left(\left(\chi_{i} \circ \psi_{i}^{-1}\right) h_{\lambda}\right) \circ \psi_{i}\right\|_{H(1 / 2)} \\
& \leq C_{i}\left\|\left(\chi_{\imath} \circ \psi_{i}^{-1}\right) h_{\lambda}\right\|_{H(1 / 2)} .
\end{aligned}
$$

Now $\chi_{i} \circ \psi_{i}^{-1}$ has compact support, so choosing some function $\varrho \in \mathcal{D}(\mathbf{R})$ with $\varrho \equiv 1$ on a large enough interval, we find that

$$
\left(\chi_{i} \circ \psi_{i}^{-1}\right) h_{\lambda}=\left(\chi_{i} \circ \psi_{i}^{-1}\right)(\varrho \otimes \varrho \otimes \varrho) h_{\lambda},
$$

and hence

$$
\begin{aligned}
\left\|\left(\chi_{i} \circ \psi_{i}^{-1}\right) h_{\lambda}\right\|_{H(1 / 2)} & \leq c_{i}\left\|(\varrho \otimes \varrho \otimes \varrho) h_{\lambda}\right\|_{H(1 / 2)} \\
& \leq c_{i}\|\varrho \otimes \varrho\|_{H_{2}(1 / 2)} \gamma_{i}\left\|h_{\lambda}\right\|_{H_{1}(1 / 2)}
\end{aligned}
$$

for some positive numbers $c_{i}$ and $\gamma_{i}$, where $H_{1}(1 / 2)$ and $H_{2}(1 / 2)$ are the analogues of $H(1 / 2)$ in one and two dimensions, respectively, and we have used the continuity of the multiplication again (cf. Appendix E.3) and also that

$$
k_{1}^{2}+k_{2}^{2}+k_{3}^{2}+1 \leq\left(k_{1}^{2}+k_{2}^{2}+1\right)\left(k_{3}^{2}+1\right)
$$

for all $k_{j} \in \mathbf{R}$. So the proof is completed by observing that

$$
\left(\left\|h_{\lambda}\right\|_{H_{1}(1 / 2)}\right)^{2}=\int|\hat{h}(k)|^{2} \lambda \sqrt{\left(\lambda^{-1} k\right)^{2}+1} d k
$$

is uniformly bounded for $\lambda \in(0,1)$.

\section{Appendix E}

E.1. We summarize some results which we use in the second part of the proof of Theorem 3.9.

Let $\mathcal{B}$ be a $\mathcal{C}^{*}$-algebra and $\pi: \mathcal{B} \rightarrow B(\mathcal{H})$ a representation of $\mathcal{B}$ by bounded linear operators on a complex Hilbertspace $\mathcal{H}$. If some closed subspace $\mathcal{K}$ of $\mathcal{H}$ is left invariant by all $\pi(A), A \in \mathcal{B}$, then $\pi$ possesses a subrepresentation $\pi^{\prime}$, defined by

$$
\pi^{\prime}(A):=\pi(A) \mid \mathcal{K} \quad \forall A \in \mathcal{B} .
$$

With this notation, we quote the following theorem (cf. Proposition 5.3.5 in [Dix]):

If the von Neumann algebra $\pi(\mathcal{B})^{\prime \prime}$ is a factor, then every subrepresentation $\pi^{\prime}$ of $\pi$ for which $\mathcal{K} \neq\{0\}$ is quasiequivalent to $\pi$.

Now let $\tilde{\mathcal{B}}$ be a sub- $C^{*}$-algebra of $\mathcal{B}$, and $\omega$ a state of $\mathcal{B}$ with GNS-representation $\left(\mathcal{H}_{\omega}, \pi_{\omega}, \Omega_{\omega}\right)$. Let $\tilde{\omega}:=\omega \mid \tilde{\mathcal{B}}$, and denote by $\left(\mathcal{H}_{\tilde{\omega}}, \pi_{\tilde{\omega}}, \Omega_{\tilde{\omega}}\right)$ its GNS-representation. Then $\pi_{\tilde{\omega}}$ can be viewed as a subrepresentation of the representation

$$
\pi_{\omega} \mid \tilde{\mathcal{B}}: \tilde{\mathcal{B}} \rightarrow \mathcal{B}\left(\mathcal{H}_{\omega}\right)
$$


To see this, recall that we have for all $A \in \tilde{\mathcal{B}}$,

$$
\tilde{\omega}(A)=\left\langle\Omega_{\tilde{\omega}}, \pi_{\tilde{\omega}}(A) \Omega_{\tilde{\omega}}\right\rangle=\left\langle\Omega_{\omega},\left(\pi_{\omega} \mid \tilde{\mathcal{B}}\right)(A) \Omega_{\omega}\right\rangle .
$$

So if we denote by $\mathcal{K}$ the closed subspace of $\mathcal{H}_{\omega}$ which is spanned by all vectors of the form $\left(\pi_{\omega} \mid \tilde{\mathcal{B}}\right)(A) \Omega_{\omega}, A \in \tilde{\mathcal{B}}$, then, by the characterizing properties of the GNS-representation, $\left(\mathcal{H}_{\tilde{\omega}}, \pi_{\tilde{\omega}}, \Omega_{\tilde{\omega}}\right)$ can be identified with $\left(\mathcal{K}, \pi_{\omega} \mid \tilde{\mathcal{B}}, \Omega_{\omega}\right)$.

Now consider two states $\omega_{j}, j=1,2$, on $\mathcal{B}$, with GNS-representations $\pi_{\omega_{j}}$, and restrictions $\tilde{\omega}_{j}:=\omega_{j} \mid \tilde{\mathcal{B}}$ to $\tilde{\mathcal{B}}$, whose GNS-representations we denote by $\tilde{\pi}_{j}$. If the $\pi_{\omega_{j}}(\tilde{\mathcal{B}})^{\prime \prime}$ are factors, then, by the just quoted theorem, and since the $\tilde{\pi}_{j}$ are canonically identifiable with subrepresentations of the $\pi_{\omega_{j}} \mid \tilde{\mathcal{B}}$, it can now easily be seen that the following holds:

The $\pi_{\omega_{j}} \mid \tilde{\mathcal{B}}$ are quasiequivalent if, and only if, the $\tilde{\pi}_{j}$ are quasiequivalent.

E.2. Here we quote the following specialization of a result by Heinz, which is stated and proved as Theorem 9.3 in [Wei] (however, we refer true connoisseurs of "operator monotony" to the original article [Hei]):

Let $\mathcal{H}$ be a Hilbert space, $T$ selfadjoint, non-negative operator and $S$ a symmetric operator in $\mathcal{H}$ with $\operatorname{dom}(T) \subset \operatorname{dom}(S)$ and $\|S f\| \leq\|T f\|$ for all $f \in \operatorname{dom}(T)$. Then

$$
|\langle f, S f\rangle| \leq\langle f, T f\rangle \quad \forall f \in \operatorname{dom}(T) .
$$

Observe that this result immediately generalizes to the case that $T$ is essentially selfadjoint, for which we employ it.

E.3. We quote a simplified version of Theorem 2.2.5 in [Hör] (to which we refer for a proof) which states that multiplication by smooth functions with compact support is a continuous operation on Sobolov spaces.

Let $n \in \mathbf{N}, m \in \mathbf{R}$, and denote by $H_{n}(m)$ the completion of $\mathcal{D}_{\mathbf{C}}\left(\mathbf{R}^{n}\right)$ in the norm given by

$$
\left(\|f\|_{H_{n}(m)}\right)^{2}:=\int_{\mathbf{R}^{n}}|\hat{f}(k)|^{2}\left(|k|^{2}+1\right)^{m} d^{n} k \quad \forall f \in \mathcal{D}_{\mathbf{C}}\left(\mathbf{R}^{n}\right),
$$

where $\hat{f}$ is the Fourier-transform of $f$. Then for each $h \in \mathcal{D}_{\mathbf{C}}\left(\mathbf{R}^{n}\right)$ there is a constant $c_{h}>0$ such that

$$
\|h f\|_{H_{n}(m)} \leq c_{h}\|f\|_{H_{n}(m)} \quad \forall f \in H_{n}(m) .
$$

E.4. In the notation introduced in E.3 we quote here, again in a simplified form, Theorem 2.6.1 in [Hör], stating that the composition with diffeomorphisms is a continuous operation on Sobolev spaces.

Let $\psi$ be a diffeomorphism of an open set $\Omega_{1} \subset \mathbf{R}^{n}$ onto another open set $\Omega_{2} \subset \mathbf{R}^{n}$, and $\bar{U} \subset \Omega_{2}$. Then for each fixed $m$ there is a $C>0$ such that

$$
\|h \circ \psi\|_{H_{n}(m)} \leq C\|h\|_{H_{n}(m)} \quad \forall h \in \mathcal{D}_{\mathbf{C}}(U) .
$$




\section{References}

[Ara] Araki, H.: A lattice of von Neumann algebras associated with the quantum theory of a free Bose field. J. Math. Phys. 4, 1343-1362 (1963)

[AY] Araki, H., Yamagami, S.: On quasi-equivalence of quasifree states of the canonical commutation relations. Publ. RIMS, Kyoto Univ. 18, 283-338 (1982)

[Ban] Bannier, U.: On generally covariant quantum field theory and generalized causal and dynamical structures. Commun. Math. Phys. 118, 163-170 (1988)

[Buc] Buchholz, D.: Product states for local algebras. Commun. Math. Phys. 36, 287-304 (1974)

[BW] Baumgärtel, H., Wollenberg, M.: Causal nets of operator algebras. Berlin: Akademie Verlag 1992

[CFKS] Cycon, H.L., Froese, R.G., Kirsch, W., Simon, B.: Schrödinger operators. Berlin, Heidelberg, New York: Springer 1987

[Che] Chernoff, P.R.: Essential self-adjointness of powers of generators of hyperbolic equations. J. Funct. Anal. 12, 401-414 (1973)

[D] Dieudonné, J.: Treatise on analysis. Vol. III. New York: Academic Press 1972

[Die] Dieckmann, J.: Cauchy-surfaces in globally hyperbolic spacetime. J. Math. Phys. 29, 578 (1988)

[Dim] Dimock, J.: Algebras of local observables on a manifold. Commun. Math. Phys. 77, 219-228 (1980)

[Dix] Dixmier, J.: Les $C^{*}$-algèbres et leurs représentations. Paris: Gauthier-Villars, 1964

[FH1] Fredenhagen, K., Haag, R.: Generally covariant quantum field theory and scaling limits. Commun. Math. Phys. 108, 91-115 (1987)

[FH2] Fredenhagen, K., Haag, R.: On the derivation of Hawking radiation associated with the formation of a black hole. Commun. Math. Phys. 127, 273-284 (1990)

[FNW] Fulling, S.A., Narcowich, F.J., Wald, R.M.: Singularity structure of the two-point function in quantum field theory in curved spacetime. II. Ann. Phys. (N.Y.) 136, 243-272 (1981)

[Fri] Friedlander, F.G.: The wave equation on a curved spacetime. Cambridge: Cambridge University Press 1975

[FSW] Fulling, S.A., Sweeny, M., Wald, R.M.: Singularity structure of the two-point function in quantum field theory in curved spacetime. Commun. Math. Phys. 63, 257-264 (1978)

[Ful] Fulling, S.A.: Aspects of quantum field theory in curved spacetime. Cambridge: Cambridge University Press 1989

[Gar] Garabedian, P.R.: Partial differential equations. New York: Wiley 1964

[GK] Gonnella, G. Kay, B.S.: Can locally Hadamard quantum states have non-local singularities? Class. Quantum Gravity 6, 1445-1454 (1989)

[H1] Haag, R.: Quantum physics and gravitation. In: Velo, G., Wightman, A.S. (eds.): Constructive quantum field theory II. New York: Plenum Press 1990

[H2] Haag, R.: Local quantum physics. Berlin: Springer 1992

[Haw] Hawking, S.W.: Particle creation by black holes. Commun. Math. Phys. 43, 199-220 (1975)

[Hei] Heinz, E.: Beiträge zur Störungstheorie der Spektralzerlegung. Math. Ann. 123, 415-438 (1951)

[HNS] Haag, R., Narnhofer, H., Stein, U.: On quantum field theory in gravitational background. Commun. Math. Phys. 94, 219-238 (1984)

[Hör] Hörmander, L.: Linear partial differential operators. Berlin: Springer 1969

[Kay1] Kay, B.S.: Linear spin-zero quantum fields in external gravitatonal and scalar fields. Commun. Math. Phys. 62, 55-70 (1978)

[Kay2] Kay, B.S.: In: Proc. 10th Inter. Conf. on General Relativity and Gravitation (Padova, 1983), eds. B. Bertotti et al. (Reidel, Dordrecht, 1984), talk (see Workshop Chairman's report by A. Ashtekar, pp. 453-456); see also: Kay, B.S.: Casimir effect in quantum field theory. Phys. Rev. D 20, 3052-62 (1979)

[Kay3] Kay, B.S.: The double-wedge algebra for quantum fields on Schwarzschild and Minkowski spacetimes. Commun. Math. Phys. 100, 57-81 (1985)

[Kay4] Kay, B.S.: A uniqueness result for quasifree KMS states. Helv. Phys. Acta 58, 1017-1029 (1985) 
[Kay5] Kay, B.S.: Quantum field theory in curved spacetime. In: Bleuler, K., Werner, M. (eds.): Differential geometric methods in theoretical physics. Dordrecht: Kluwer Acadamic Publishers 1988

[KW] Kay, B.S., Wald, R.M.: Theorems on the uniqueness and thermal properties of stationary, nonsingular, quasifree states on spacetimes with a bifurcate Killing horizon. Phys. Rep. 207, 49-136 (1991)

[LR] Lüders, C., Roberts, J.E.: Local quasiequivalence and adiabatic vacuum states. Commun. Math. Phys. 134, 29-63 (1990)

[LRT] Leyland, P., Roberts, J.E., Testard, D.: Duality for quantum free fields. Preprint, Marseille (1978)

[MV] Manuceau, J., Verbeure, A.: Quasifree states of the CCR-algebra and Bogoliubov transformations. Commun. Math. Phys. 9, 293-302 (1968)

[Rad] Radzikowsi, M.J.: The Hadamard condition and Kay's conjecture in (axiomatic) quantum field theory on curved space-time. Doctoral Dissertation, Princeton University, 1992

[RS] Reed, M., Simon, B.: Methods of modern mathematical physics. II. New York: Academic Press 1975

[S] Schwartz, L.: Théorie des distributions. Paris: Hermann 1957

[Wal1] Wald, R.M.: The back reaction effect in particle creation in curved spacetime. Commun. Math. Phys. 54, 1-19 (1977)

[Wal2] Wald, R.M.: Trace anomaly of a conformally invariant quantum field in curved spacetime. Phys. Rev. D 17, 1477-84 (1978)

[Wal3] Wald, R.M.: General relativity. Chicago: University of Chicago Press 1984

[Wei] Weidmann, J.: Linear operators in Hilbert spaces. Berlin, Heidelberg, New York: Springer 1980

[Wol] Wollenberg, M.: Scaling limits and type of local algebras over curved spacetime. In: Arveson, W.B. et al. (eds.): Operator algebras and topology, Pitman Research Notes in Mathematics Series 270. Harlow: Longman 1992

Communicated by $\mathrm{H}$. Araki 